\title{
ENDOTRIVIAL MODULES FOR FINITE GROUP SCHEMES
}

\author{
JON F. CARLSON AND DANIEL K. NAKANO
}

\begin{abstract}
It is well known that if $G$ is a finite group then the group of endotrivial modules is finitely generated. In this paper we investigate endotrivial modules over arbitrary finite group schemes. Our results can be applied to computing the endotrivial group for several classes of infinitesimal group schemes which include the Frobenius kernels of parabolic subgroups, and their unipotent radicals (for reductive algebraic groups). For $G$ reductive, we also present a classification of simple, induced/Weyl and tilting modules ( $G$-modules) which are endotrivial over the Frobenius kernel $G_{r}$ of $G$.
\end{abstract}

\section{INTRODUCTION}

Let $A$ be a finite dimensional cocommutative Hopf algebra over a field $k$. An endotrivial module is an $A$-module $M$ with the property that $\operatorname{Hom}_{k}(M, M) \cong k \oplus P$ where $P$ is a projective $A$-module and the isomorphism is a map of $A$-modules. Because $\operatorname{Hom}_{k}(M, M) \cong M \otimes_{k} M^{*}$ where $M^{*}$ is the $k$-dual of $M$, we have that $M \otimes-$ is a self equivalence of the stable category of all $A$-modules modulo projective modules. Thus the endotrivial modules determine a subgroup of the Picard group of self equivalences of the stable category. In addition, endotrivial modules form an interesting class of modules which in many cases is classifiable even though the category of all $A$-modules is wild, in general.

In the case that $A=k G$ is the group algebra of a finite group $G$ with coefficients in a field of characteristic $p>0$, the endotrivial modules play a big part in the representation theory. When $G$ is a $p$-group, the first author and Thévenaz classified [15, 16] the endotrivial modules, building on the work of Dade, Alperin and others [1, 17, 18]. This work was used by Bouc [6] in his classification of endopermutation modules for $p$-groups. For other finite groups, the two authors together with Mazza and Hemmer have computed the group of endotrivial modules for groups of Lie type [12] and for alternating and symmetric groups [13, 9].

The aim of this paper is to initiate an investigation of endotrivial modules for arbitrary finite group schemes. We are particularly interested in the case where the group scheme is the Frobenius kernel of an algebraic group. Unfortunately, many of the methods used in the classification of endotrivial modules over finite groups do not work in the more general setting or can be applied only after some adaptation. For arbitrary finite group schemes, we must rely on more geometric techniques. The

Date: November 11, 2018.

1991 Mathematics Subject Classification. 20C20.

Research of the first author partially supported by NSF grant DMS-0654173.

Research of the second author partially supported by NSF grant DMS-0654169. 
methods work best in the case that the group scheme is unipotent or has a nontrivial unipotent radical. The unipotent group schemes are analogous to $p$-groups in the realm of finite groups and here we can call upon the classification by Dade of the endotrivial modules over abelian p-groups [17, 18] (even though the Hopf algebra structures may be different). In the case of a unipotent restricted $p$-Lie algebra (associated to a reductive algebra group), we obtain a complete classification of the endotrivial modules. In all of the examples that we have been able to work out, the group of endotrivial modules is finitely generated. Yet, for general finite group schemes, a proof of the finite generation of the group of endotrivial modules remains an open problem (see Section 9).

We briefly describe the results of the paper. First, we set up the necessary notation that will be used throughout the paper in the next section. In Section 3, we present general results on endotrivial modules for finite group schemes and show that if $G$ is a finite unipotent group scheme then for any fixed finite integer $n$ there is only a finite number of endotrivial $G$-modules of dimension $n$ (cf. Theorem 3.5). This indicates that for unipotent finite group schemes the problem of classifying endotrivial modules is not a wild problem. Furthermore in Section 4, for unipotent group schemes, a criterion is given via the connectedness of a subvariety of $\pi$-points, $\Pi(G)$, which is sufficient to prove that the group of endotrivial modules is isomorphic to $\mathbb{Z}$ (i.e., the endotrivial modules are given by syzygies of the trivial module). In that section, we have attempted to extract the exact hypotheses necessary to prove this result in the hopes that the methods will have wider applications.

A semisimple algebraic group scheme $G$ has a Borel subgroup $B$ with unipotent radical $U$ and, for $J$ a subset of the simple roots, parabolic subgroups $P_{J}$ and their associated unipotent radicals $U_{J}$. Let $G_{r}, B_{r},\left(P_{J}\right)_{r}$ and $\left(U_{J}\right)_{r}$ denote their $r^{\text {th }}$ infinitesimal Frobenius kernels. In Sections 5 and 6 , we compute the endotrivial groups for $B_{1}, U_{1},\left(P_{J}\right)_{1}$ and $\left(U_{J}\right)_{1}$ when $J \neq \varnothing$. We show, using the methods of Section 4 , that except in a couple of low rank cases the group of endotrivial modules for $U_{1}$ is generated by $\Omega(k)$, the syzygy of the trivial module. From this we can compute the endotrivial group for $B_{1}$ and for $\left(P_{J}\right)_{1}$. The lone remaining case for the first Frobenius kernel is the computation of the endotrivial group for $G_{1}$.

For $G=S L_{2}$ we compute the endotrivial group for the first Frobenius kernel in Section 7, and prove that there are Weyl modules (which are not syzygies of the trivial module) which are endotrivial over $G_{r}$ when $r \geq 1$. This leads us to investigate the question of classifying simple, induced/Weyl, and tilting modules over $G$ which become endotrivial over $G_{r}$. In Section 8, we demonstrate that this cannot happen when the Lie rank of $G$ is greater than two. We suspect for large enough Lie rank that the group of endotrivial modules over $G_{r}$ is isomorphic to $\mathbb{Z}$. In Section 9, we describe connections with the work of Balmer on the endotrivial group and the Picard group of the projectivization of the cohomological spectrum. In the final section, we present some additional open problems that are inspired from the results of this paper.

The authors would like to thank Ivo Dell'Ambrogio and Robert Varley for several useful discussions involving Picard groups and their connections with endotrivial modules. 


\section{Notation AND PRELIMINARIES}

In this section we set some notation and recall a few facts about representations of group schemes. Throughout the paper, let $k$ be a field of characteristic $p>0$. A finite group scheme $G$ over $k$ is a group scheme over $k$ whose coordinate algebra $k[G]$ has finite $k$-dimension. The group algebra of $G$ is the dual of $k[G]$, the coordinate ring, and is denoted $k G$. The algebra $k G$ is a cocommutative Hopf algebra. In some cases $k G$ is isomorphic as an algebra to the group algebra of a finite group. For example, if $G$ is a unipotent abelian group scheme, then $k G$ is isomorphic as an algebra to the group algebra of a finite abelian $p$-group, though the coalgebra structures may be different.

By a $k G$-module we mean a finitely generated $k G$-module. In particular, we need to define the rank variety for a $k G$-module in terms of $\pi$-points as in the work of Friedlander and Pevtsova [22]. Some of the notation that is introduced in this section is mostly necessary for the definitions of the rank variety.

A rational $G$-module is both a module for the group algebra $k G$ and comodule for the coordinate ring $k[G]$. For a field extension $K$ of $k$ let $G_{K}$ be the base change of the $k$-group scheme $G$ to the $K$-group scheme $G_{K}=G \times_{\operatorname{Spec}(k)} \operatorname{Spec}(K)$. Note that that the group algebra $K G_{K}$ of $G_{K}$ is the extension of scalars, $K G=K \otimes_{k} k G$.

A $\pi$-point for a finite group scheme $G$ is a flat map of $K$-algebras $\alpha_{K}: K[t] /\left(t^{p}\right) \longrightarrow$ $K G_{K}$, where $K$ is a field extension of $k$, such that the map factors through the group algebra $K U_{K} \subset K G_{K}$ of some unipotent abelian subgroup scheme $U_{K} \subset G_{K}$. A $\pi$-point is not assumed to be a map of Hopf algebras.

To define the rank variety of $\pi$-points we must introduce an equivalence relation. We say that two $\pi$-points $\alpha_{K}, \beta_{L}$ are equivalent if for every finitely generated $k G$ module $M$, the $K[t] /\left(t^{p}\right)$-module $\alpha_{K}^{*}\left(M_{K}\right)$ is projective if and only if the $L[t] /\left(t^{p}\right)$ module $\beta_{L}^{*}\left(M_{L}\right)$ is projective. Here $\alpha_{K}^{*}\left(M_{K}\right)$ is the restriction of $M_{K}=K \otimes M$ to a $K[t] /\left(t^{p}\right)$-module along the map $\alpha_{K}$.

The set of equivalence classes of $\pi$-points, which we denote by $\Pi(G)$, has a scheme structure which is defined by the representation theory. That is, a subset of $\Pi(G)$ is defined to be closed if it has the form $\Pi(G)_{M}$ where $M$ is a finite dimensional $k G$-module and $\Pi(G)_{M}$ is the subset of those equivalence classes of $\pi$-points $\alpha_{K}: K[t] /\left(t^{p}\right) \longrightarrow K G$ such that $\alpha_{K}^{*}\left(M_{K}\right)$ is not a projective module. With this definition, it can be proved that the scheme $\Pi(G)$ is homeomorphic to the scheme $\operatorname{Proj}\left(\mathrm{H}^{*}(G, k)\right)$, the projective prime ideal spectrum of $\mathrm{H}^{*}(G, k)$.

In the case that the field $k$ is algebraically closed we can get a simpler formulation which is called the set of $p$-points [21]. A $p$-point is simply a $\pi$-point $\alpha: k[t] /\left(t^{p}\right) \longrightarrow$ $k G$ which involves no field extension. Two $p$-points are equivalent if they satisfy the same condition as above, i.e., for any $k G$-module $M$, the restriction along one is projective if and only if the restriction along the other is projective. We also impose a topology on $\pi(G)$, the set of equivalence classes of $p$-points, exactly as above. With this topology, for $k$ algebraically closed $\pi(G)$ is homeomorphic to the projectivized maximal ideal spectrum of $\mathrm{H}^{*}(G, k)$. This is the variety of closed points in $\operatorname{Proj}\left(\mathrm{H}^{*}(G, k)\right)$. A $k G$-module $M$ is projective if and only if its restriction along every $p$-point is projective. 
Modules over the algebra $k[t] /\left(t^{p}\right)$ are classified by Jordan type, i.e., by the Jordan canonical form of the matrix $A_{t}$ of the action of the $t$ on the module. Because, $\left(A_{t}\right)^{p}=0$, we know that all of the eigenvalues of $A_{t}$ are 0 and no Jordan block has more than $p$ rows. We write that the Jordan type of a $k[t] /\left(t^{p}\right)$-module $M$ is $a_{p}[p]+\cdots+a_{1}[1]$ if the matrix $A_{t}$ of $t$ on $M$ has $a_{p}$ blocks of size $p, a_{p-1}$ blocks of size $p-1$, etc. Here,

$$
a_{p} p+a_{p-1}(p-1)+\cdots+a_{1}=n=\operatorname{dim} M .
$$

The Jordan type is a partition of $n=\operatorname{dim} M$ having the form $\left(p^{a_{p}},(p-1)^{a_{p-1}}, \ldots, 1^{a_{1}}\right)$, where for each $i$ there are $a_{i}$ of the entries in the partition with value $i$.

The next result is well known, but we sketch a proof for the sake of completeness.

Lemma 2.1. Suppose that $G$ is a finite unipotent group scheme over $k$. Then the socle of $k G$ has dimension one. Let $u$ be a generator for the socle of $k G$. Suppose that $M$ is a $k G$-module and that $u M$ has dimension $r$. Then $M \cong(k G)^{r} \oplus N$ where $N$ has no projective submodules.

Proof. The first statement is a consequence of the fact that $k G$ is a local self-injective $k$-algebra with only a single isomorphism class of irreducible modules. In particular, we note that every projective $k G$-module is free. The reader is referred to Chapter 8 in Part I of [26] for more details. Let $u$ be a generator for the socle and suppose that $M$ is a finitely generated $k G$-module. Let $u m_{1}, u m_{2}, \ldots, u m_{r}$ be a basis of $u M$ for some elements $m_{1}, \ldots, m_{r} \in M$. Let $P$ be a free $k G$-module of $k G$-rank $r$ having generators $e_{1}, \ldots, e_{r}$. Then the map $\psi: P \longrightarrow M$, given by $\psi\left(e_{i}\right)=m_{i}$ for $i=1, \ldots, r$ is an injection, because it is injective on the socle of $P$. But now, $P$ is also an injective module and hence $\psi$ is left split. So, $M \cong P \oplus N$ for some $N$. We know that $N$ has no projective submodules because $u N=\{0\}$.

\section{Generalities on endotrivial modules}

In this section we consider some basic issues with endotrivial modules over a unipotent group scheme $k G$. The results here will be important in the remainder of the paper. Throughout the section we assume that the field $k$ is algebraically closed. First the definition.

Definition 3.1. Suppose that $G$ is any finite group scheme defined over $k$. A $k G$ module is an endotrivial module provided that, as $k G$-modules,

$$
\operatorname{Hom}_{k}(M, M) \cong k \oplus P
$$

for some projective module $P$.

In other words, a $k G$-module $M$ is an endotrivial module if its $k$-endomorphism ring is isomorphic to the trivial module in the stable category of $k G$-modules modulo projectives. For any $k G$-module $M$ there is a canonical isomorphism $\operatorname{Hom}_{k}(M, M) \cong$ $M^{*} \otimes M$. Using this we define the group $T(k G)$ of endotrivial $k G$-modules as follows. The objects in $T(k G)$ are the equivalence classes $[M]$ of endotrivial $k G$-modules $M$ where the equivalence relation is given by the rule that $[M]=[N]$ if there are projective modules $P$ and $Q$ such that $M \oplus P \cong N \oplus Q$. The operation on the 
group is given by $[M]+[N]=[M \otimes N]$. Hence, the identity element is the class $[k]$ and the inverse of $[M]$ is the class $\left[M^{*}\right]$.

The following theorem proved in 8 is very useful for our analysis. Note that the Jordan type of a $k G$-module is completely independent on the coalgebra structure. Consequently, the theorem implies that the property of a $k G$-module being endotrivial is independent of the coalgebra structure. We emphasize that this result depends on the field $k$ being algebraically closed. Otherwise, we must rely on $\pi$-points rather than $p$-points.

Theorem 3.2. Suppose that $G$ is a finite group scheme defined over $k$ and that $M$ is a finitely generated $k G$-module. Then $M$ is an endotrivial module if and only if for any p-point $\alpha_{k}: k[t] /\left(t^{p}\right) \longrightarrow k G$, the restriction $\alpha^{*}(M)$ has Jordan type either $[1]+n[p]$ or $[p-1]+n[p]$ for some integer $n$.

Proof. We note first that if $M$ is endotrivial then the Jordan type of any $p$-point is as stated. Hence, we need only prove the converse. We know from Theorem 5.6 of [8], that $M$ is endotrivial if $\alpha_{K}^{*}\left(M_{K}\right)$ has the prescribed Jordan type for all $\pi$-points $\alpha_{K}: K[t] /\left(t^{p}\right) \longrightarrow K G_{K}$. We need only show that $M$ is endotrivial if the condition holds for $\pi$-points defined over $K=k$.

Suppose that for any $p$-point $\alpha: k[t] /\left(t^{p}\right) \longrightarrow k G$ the restricted module $\alpha^{*}(M)$ has Jordan type either $[1]+n[p]$ or $[p-1]+n[p]$ for some integer $n$. Then the partition corresponding to this Jordan type is maximal in the dominance ordering of partitions of $\operatorname{dim}(M)$. Hence, such a $p$-point has maximal Jordan type. By [23], any $\pi$-point in the equivalence class of such an $\alpha$ also has maximal Jordan type. Any $\pi$-point $\alpha_{K}: K[t] /\left(t^{p}\right) \longrightarrow K G$, must also have maximal Jordan type $[1]+n[p]$ or $[p-1]+n[p]$. The reason is that, because $k$ is algebraically closed and $M$ is finite dimensional, the class of $\alpha_{K}$ must specialize to some closed point $[\beta]$ in $\Pi(G)$ and the Jordan type of $\alpha_{K}^{*}\left(M_{K}\right)$ must be larger than that of $\beta^{*}(M)$. As the closed points in $\Pi(G)$ are represented by $p$-points defined over $k$, the Jordan type of $\alpha_{K}^{*}\left(M_{K}\right)$ must be maximal. We conclude that $M$ has constant Jordan as defined in [8], and by the first statement of the theorem, $M$ is endotrivial.

An immediate application of the theorem is the following.

Lemma 3.3. Suppose that $G$ is a unipotent abelian group scheme. Then any endotrivial $k G$-module is isomorphic to $\Omega^{n}(k) \oplus Q$ for some $n$ and some projective module $Q$.

Proof. The group algebra $k G$ is isomorphic to $k E$ where $E$ is an abelian $p$-group. This is an isomorphism as algebras, but not as Hopf algebras. By Theorem [3.2, a $k G$-module or a $k E$-module is endotrivial if and only if it has constant Jordan type $[1]+n[p]$ or $[p-1]+n[p]$ for some $n$. Consequently, any endotrivial $k G$-module is also an endotrivial $k E$-module and hence is isomorphic to $\Omega^{n}(k) \oplus Q$ for some some $n$ and some projective module $Q$, by the theorem of Dade [17, 18].

Another consequence of the theorem is the following result on restrictions. 
Proposition 3.4. Suppose that $G$ and $H$ are finite group schemes defined over $k$ and suppose that we have a flat map $\gamma: k H \rightarrow k G$. If $M$ is an endotrivial $k G$-module, then the pullback along $\gamma$ of $M$ is an endotrivial $k H$-module.

Proof. Observe that regardless of the coalgebra structure on $k H$, any $\pi$-point for $H$ becomes a $\pi$-point for $G$ when composed with the inclusion map $k H \rightarrow k G$. Consequently, $\left.M\right|_{k H}$ has constant Jordan type exactly as required for an endotrivial module by Theorem 3.2 .

The next result follows the lines of a idea of Dade [19], but for the sake of completeness we present a detailed proof. The result is not as strong as what we can get in the case of a group algebra. That is, it does not follow automatically that the group of endotrivial modules is finitely generated.

Theorem 3.5. Suppose that $G$ is a finite unipotent group scheme. For any positive integer $n$, there is at most a finite number of endotrivial $k G$-modules of dimension $n$.

Proof. We wish to consider the variety $\mathcal{V}_{n}$ consisting of all representations of $k G$ of dimension $n$. It can be described as follows. Suppose that $g_{1}, g_{2}, \ldots, g_{t}$ is a set of generators of the algebra $k G$, so that every element of $k G$ can be written as a polynomial in (noncommuting) variables $g_{1}, \ldots, g_{t}$. Thus, $k G \cong k\left\langle g_{1}, \ldots, g_{t}\right\rangle / \mathcal{J}$ for some ideal $\mathcal{J}$.

The variety $\mathcal{V}_{n}$ is defined to be the zero locus in $k^{t n^{2}}$ determined by the ideal $\mathcal{J}$ of relations on the generators $g_{i}$. Specifically, the construction is the following. A representation of $k G$ of dimension $n$ is a homomorphism $\varphi: k G \longrightarrow M_{n}$ where $M_{n}$ is the algebra of $n \times n$ matrices over $k$. So for each $i$, the image of $g_{i}$ is a matrix $\varphi\left(g_{i}\right)=\left(a_{j, \ell}^{i}\right)$. In the general representation, we can consider elements $a_{j, \ell}^{i}$ to be variables or indeterminants. A relation among the generators of $k G$, gives us a collection of relations among the variables. For example, if it were the case that $g_{i} g_{j}=0$ (so that $\left.g_{i} g_{j} \in \mathcal{J}\right)$, then we would have the relations $f_{r, t}=\sum_{s=1}^{n} a_{r, s}^{i} a_{s, t}^{j}=0$ for all $1 \leq r, t \leq n$. The elements $f_{r, t}$ are then elements of an ideal $\mathcal{I} \subseteq R=k\left[a_{j, \ell}^{i}\right]$, for $1 \leq i \leq t, 1 \leq j, \ell \leq n$. The variety $\mathcal{V}_{n}$ can be taken to be the maximal ideal spectrum of the ring $R / \mathcal{I}$ or alternatively as the zero locus of $\mathcal{I}$ in $k^{t n^{2}}$. For more information on the variety $\mathcal{V}_{n}$ see the paper of Dade [19].

Now let $u$ be a generator for the socle of $k G$. Recall that by Lemma 2.1, the dimension of $u M$ is the rank of the largest projective summand of the $k G$-module $M$. Suppose that $M$ is an endotrivial $k G$-module of dimension $n$. Then $n \equiv$ $\pm 1(\bmod p)$ and $M \otimes M^{*} \cong k \oplus Q$ where $Q$ is projective module of dimension $p^{u} r$ where $p^{u}=\operatorname{dim}(k G)$ and $r$ is some positive integer. So $(\operatorname{dim}(M))^{2}=1+\operatorname{dim}(Q)$.

Now we choose a particular representation of $\rho: k G \longrightarrow M_{n}$ of the dual module $M^{*}$. Let $\mathcal{S}$ be the set of all subsets of $\mathfrak{N}=\{1, \ldots, n\}$ having exactly $r$ elements. For any pair $S, T$ of elements of $\mathcal{S}$ define $f_{S, T}: \mathcal{V}_{n} \longrightarrow k$ by the rule that $f_{S, T}(\gamma)=$ $\operatorname{Det}\left(M_{S, T}((\gamma \otimes \rho)(u))\right)$. Here $(\gamma \otimes \rho)(u)$ is the action matrix of $u$ on the module $N \otimes M^{*}$ given by the tensor product of the representations $\gamma \otimes \rho$ where $N$ is the module affording the representation $\gamma$. Also $M_{S, T}$ is the $r \times r$ submatrix whose rows 
are indexed by the elements of $S$ and whose columns are indexed by the elements of $T$. Finally, Det is the determinant function.

The important item to notice is that each $f_{S, T}$ is a polynomial map. Specifically, any entry of the matrix $(\gamma \otimes \rho)(v)$ is a polynomial in the entries of $\gamma(v)$ (remember $\rho$ and hence $\rho(v)$ are fixed) for generators $v$ of $k G$. Then $u$ is a polynomial in the generators of $k G$. Also, Det is a polynomial function in the entries of the matrix.

The result of this is that the zero locus of $f_{S, T}$ is a closed set in $\mathcal{V}_{n}$. Let $W_{M}$ be the closed set which is the intersection of the zero loci of all $f_{S, T}$ for all $S, T \in \mathcal{S}$. We claim that if $\gamma \in \mathcal{V}_{n}$ is not in $W_{M}$, then the module $N$ afforded by $\gamma$ is isomorphic to $M$. The reason for this is that if $\gamma \notin W_{M}$, then the rank of $u$ on $N \otimes M^{*}$ is $r$. Therefore $N \otimes M^{*}$ has a direct summand isomorphic to $k G^{r}$. The only way this could happen is if $N \otimes M^{*} \cong k \oplus k G^{r}$, by a dimension argument. Hence $[N]=[M]$ as desired.

The result we have proved says that if $M$ is an endotrivial $k G$-module then the set of all representations isomorphic to $M$ is an open set in $\mathcal{V}_{n}$ whose closure must then be a component of the variety $\mathcal{V}_{n}$. Since the variety has only a finite number of components, the theorem is proved.

\section{Unipotent Finite GROUP SCHEMES With PBW BASES.}

Throughout this section let $k$ be an algebraically closed field of characteristic $p>0$. In this section we wish to consider finite group schemes satisfying the following hypotheses.

Hypothesis 4.1. We assume that $G$ is a finite unipotent group scheme having the property that the group algebra $A=k G$ has a set of generators $u_{1}, \ldots, u_{n}, n>1$, with the properties that

(a) for each $i=1, \ldots, n, u_{i}^{p}=0$,

(b) for each $i=1, \ldots, n-1$, the class of element $u_{i}$ is central in $k G / I_{i}$ where $I_{i}$ is the ideal generated by $u_{i+1}, \ldots, u_{n}$,

(c) the element $u_{n}$ is central in $A$, and

(d) the dimension of $A$ is $p^{n}$.

An example of such a group algebra is the restricted enveloping algebra $u(\mathfrak{n})$ of a unipotent restricted $p$-Lie algebra $\mathfrak{n}$ such that $x^{[p]}=0$ for every $x \in \mathfrak{n}$. Such a Lie algebra has a basis $x_{1}, \ldots, x_{t}$ such that $x_{t}$ is central and corresponding to this is a basis for $A$ consisting of elements $u_{i}$ as in the hypothesis.

It is likely that some of the conditions of Hypothesis 4.1 can be relaxed or altered and still imply the results that we prove later in the paper. One of the things that we really need is that the algebra have a Poincaré-Birkhoff-Witt basis as in the following.

Lemma 4.2. Suppose that $A=k G$ has a collection of generators $u_{1}, \ldots, u_{n}$ satisfying the conditions of Hypothesis 4.1. Then we have the following.

(a) The algebra $A$ has a $k$-basis consisting of the set

$$
S=\left\{u_{1}^{i_{1}} u_{2}^{i_{2}} \cdots u_{n}^{i_{n}} \mid 0 \leq i_{j}<p, \quad j=1, \ldots, n\right\} .
$$


(b) For any $s=1, \ldots, n$, the ideal $J_{s}=A u_{s}+\cdots+A u_{n}$ is a two sided ideal and $J_{s}\left(u_{s}^{p-1} \cdots u_{n}^{p-1}\right)=0$. That is, if $x$ is any element of $J_{s}$, then $x u_{s}^{p-1} \cdots u_{n}^{p-1}=$ 0 .

(c) Suppose that $v=a_{1} u_{1}+\cdots+a_{n} u_{n}$ for $a_{1}, \ldots, a_{n} \in k$ and $a_{i} \neq 0$ for some $i<n$. If $v^{p}=0$, then $v^{p-1} \notin A u_{n}$, and the subalgebra $E=k\left\langle v, u_{n}\right\rangle$ generated by $v$ and $u_{n}$ is isomorphic to the group algebra of an elementary abelian $p$ group of rank 2.

Proof. The proof of the first statement is a standard argument. That is, we proceed by an induction on $n$, noting that the result is true if $n=2$ since the algebra $A$ in that case is commutative. A brief sketch of the argument goes as follows. First notice that the dimension of $A / A u_{n}$ is precisely the number of Jordan blocks of the matrix of the action of $u_{n}$ on $A$. Because none of the blocks has more than $p$ rows and the dimension of $A$ is $p^{n}$, we conclude that the dimension of $A / A u_{n}$ is at least $p^{n-1}$. Likewise the dimension of $A /\left(A u_{n-1}+A u_{n}\right)$ is the number of Jordan blocks of the matrix of the action of $u_{n-1}$ on $A / A u_{n}$. Again, we conclude that the dimension of $A /\left(A u_{n-1}+A u_{n}\right)$ is at least $p^{n-2}$. For this reason, we assume in the induction that follows, that the dimension of $A$ is at least $p^{n}$.

Now suppose that $n>2$, and by induction assume that the result is true whenever there are fewer than $n$ generators. Thus by the assumption the result holds for $A / A u_{n}$. Then any word in $u_{1}, \ldots, u_{n}$ can be rewritten as a linear combination of elements of $S$ and a linear combination of words which all involve $u_{n}$ (which we recall is central). Each of these latter words can be written as the product $w u_{n}$ where $w$ is a word in $u_{1}, \ldots, u_{n-1}, u_{n}$. Hence, by induction, it can be written as a linear combination of elements of $S$ and words which now involve $u_{n}^{2}$. Continuing this process we see that $S$ spans $A$. By a dimension argument $S$ must be a $k$-basis.

The fact that $J_{s}$ is a two sided ideal is a consequence of parts $(b)$ and $(c)$ of Hypothesis 4.1. To prove the second statement in $(b)$, we need only show that $u_{j}\left(u_{s}^{p-1} \cdots u_{n}^{p-1}\right)=0$ for any $j \geq s$. For $j=s$ this is obvious. For $j>s$ we need only note that $u_{j} u_{s}^{p-1}=u_{s}^{p-1} u_{j}+w$ for some $w$ in $J_{j+1}$ by the Hypothesis. Hence,

$$
u_{j}\left(u_{s}^{p-1} \cdots u_{n}^{p-1}\right) \in J_{s+1}\left(u_{s+1}^{p-1} \cdots u_{n}^{p-1}\right)=0
$$

by a downward induction on $s$. This proves $(b)$.

Now assume the hypothesis of $(c)$. The subalgebra $E$ is commutative, and the two generators $v$ and $u_{n}$ have the property that $v^{p}=0=u_{n}^{p}$. So there is a surjective homomorphism $\varphi: k[s, t] /\left(s^{p}, t^{p}\right) \longrightarrow E$ with $\varphi(s)=v$ and $\varphi(t)=u_{n}$. To show that this is an injection we need only show that $v^{p-1} u_{n}^{p-1} \neq 0$, since $s^{p-1} t^{p-1}$ is a $k$-basis for the minimal ideal of $k[s, t] /\left(s^{p}, t^{p}\right)$. Now we can write $v=a_{i} u_{i}+a_{i+1} u_{i+1}+\cdots+a_{n} u_{n}$ where $a_{i} \neq 0$. That is, we assume that $i$ is the first index such that $a_{i}$ is not zero. Then we have that $v^{p-1}=a_{i}^{p-1} u_{i}^{p-1}+w$ where $w \in J_{i+1}$. Then

$$
v^{p-1}\left(u_{i+1}^{p-1} \cdots u_{n}^{p-1}\right)=a_{i}^{p-1} u_{i}^{p-1} u_{i+1}^{p-1} \cdots u_{n}^{p-1} \neq 0
$$

by parts (a) and (b) of this lemma. 
In addition we require the following hypothesis in some circumstances. For notation, let $\mathcal{V}(A)$ be the set of points $\bar{a}=\left[a_{1}, \ldots, a_{n}\right]$ in projective $k$-space $\mathbb{P}^{n-1}$ such that $v=\sum_{i=1}^{n} a_{i} u_{i}$ has the property that $v^{p}=0$.

Hypothesis 4.3. Assume that $A=k G$ satisfies Hypothesis 4.1 with the given notation. We assume that every equivalence class of p-points has a representative $\alpha: k[t] /\left(t^{p}\right) \rightarrow A$, with the form $\alpha(t)=\sum_{i=1}^{n} a_{i} u_{i}$ for elements $a_{1}, \ldots, a_{n}$ such that the projective point $\bar{a}=\left[a_{1}, \ldots, a_{n}\right]$ is in $\mathcal{V}(A)$. Moreover, we assume that the association of the class of $\alpha$ to the point $\bar{a}$ induces a homeomorphism $\phi: \pi(G) \rightarrow \mathcal{V}(A)$.

Let $\hat{\mathcal{V}}(A)$ be the image of the projection

$$
\mathcal{V}(A) \backslash\{[0, \ldots, 0,1]\} \longrightarrow \mathbb{P}^{n-2}
$$

obtained by sending $\left[a_{1}, \ldots, a_{n}\right]$ to $\left[a_{1}, \ldots, a_{n-1}\right]$. We assume further that $\hat{\mathcal{V}}(A)$ is connected.

Note in the hypothesis, that the existence of the induced homeomorphism $\phi$ requires that $\phi(\alpha)=\phi(\beta)$ if $\alpha$ and $\beta$ are equivalent $p$-points. In addition, the map $\alpha: k[t] /\left(t^{p}\right) \rightarrow A$, defined by $\alpha(t)=u_{n}$ is a $p$-point and hence the element $[0, \ldots, 0,1] \in \mathbb{P}^{n-1}$ is in $\mathcal{V}(A)$.

The result of that gets us started is the following.

Theorem 4.4. Assume that $A=k G$ satisfies Hypotheses 4.1 and 4.3. Suppose that $M$ is a finitely generated endotrivial A-module. There exists a number $s$ with the property that if $\alpha: k[t] /\left(t^{p}\right) \rightarrow A$ is a p-point such that $\alpha(t)=a_{1} u_{1}+\cdots+a_{n} u_{n}$ with $a_{i} \neq 0$ for some $i<n$ and if $E=\left\langle v, u_{n}\right\rangle$ is the subalgebra of dimension $p^{2}$ generated by $v=\alpha(t)$ and $u_{n}$, then $\left.M\right|_{E} \cong \Omega^{s}(k) \oplus($ proj $)$.

Proof. By the hypothesis, any $p$-point $\alpha: k[t] /\left(t^{p}\right) \rightarrow A$, has an equivalent $p$-point such that $\alpha(t)=v_{\bar{a}}=\sum_{i=1}^{n} a_{i} u_{i}$ for some element $\bar{a}=\left[a_{1}, \ldots, a_{n}\right]$ of $\mathcal{V}(A)$. If $a_{i} \neq 0$ for some $i<n$, let $E_{\bar{a}}=\left\langle v_{\bar{a}}, u_{n}\right\rangle$ be the subalgebra generated by $v_{\bar{a}}$ and $u_{n}$. We emphasize that the class of the $p$-point does not depend on the choice of the representative. That is, the $p$-point $\beta: k[t] /\left(t^{p}\right) \rightarrow k G$ with $\beta(t)=c a_{1} u_{1}+\cdots+c a_{n} u_{n}$ is equivalent to $\alpha$ for any $c \neq 0$ in $k$. For our purposes it is important that if $a_{i} \neq 0$ for some $i<n$, then the subalgebras $E_{\bar{a}}$ and $E_{c \bar{a}}$ are the same. Moreover, $E_{\bar{a}}$ is the same as $E_{\bar{a}^{\prime}}$, where $\bar{a}^{\prime}=\left[a_{1}, \ldots, a_{n-1}\right] \in \hat{\mathcal{V}}(A)$. Therefore, for any $\bar{a} \in \hat{\mathcal{V}}(A)$. We will use the notation $v_{\bar{a}}$ and $E_{\bar{a}}$ without further explanation.

Suppose that $\bar{a}=\left[a_{1}, \ldots, a_{n-1}\right] \in \hat{\mathcal{V}}(A) \subseteq \mathbb{P}^{n-2}$. By Lemma 4.2(c), there is a $p$ point $\alpha: k[t] /\left(t^{p}\right) \rightarrow A$ such that $\alpha(t)=v_{\bar{a}}=\sum_{i=1}^{n-1} a_{i} u_{i}$. Let $E_{\bar{a}}=\left\langle v_{\bar{a}}, u_{n}\right\rangle$. As an algebra $E_{\bar{a}}$ is isomorphic to the group algebra of an elementary abelian $p$-subgroup of rank 2 by Lemma 4.2 (c).

Let $\left.M\right|_{E_{\bar{a}}}$ denote the restriction of $M$ to a module over $E_{\bar{a}}$. As such we have that

$$
\left.M\right|_{E_{\bar{a}}} \cong \Omega^{m_{\bar{a}}}(k) \oplus(\mathrm{proj})
$$

for some integer $m_{\bar{a}}$. This is a consequence of Proposition 3.4 and Lemma 3.3. Our purpose, then, is to prove that $m_{\bar{a}}$ is a constant, independent of $\bar{a}$. Note that $m_{\bar{a}}=m_{c \bar{a}}$ for any nonzero $c \in k$. 
Next we observe that there exist numbers $b$ and $B$ such that $b \leq m_{\bar{a}} \leq B$. The reason is that for $m$ sufficiently large we have

$$
\operatorname{dim} \Omega^{m}\left(\left.k\right|_{E}\right)=\operatorname{dim} \Omega^{-m}\left(\left.k\right|_{E}\right)>\operatorname{dim} M
$$

for $E \cong E_{\bar{a}}$ for any $\bar{a}$. Now choose $b$ and $B$ so that for some $a, m_{\bar{a}}=b$ and for some $\bar{a}^{\prime}, m_{\bar{a}^{\prime}}=B$. Next we replace $M$ by $\Omega^{-b}(M)$. Then we can assume that $b=0$, and for every $\bar{a} \in \hat{\mathcal{V}}(A) \subseteq \mathbb{P}^{n-2}$, we have that $0 \leq m_{\bar{a}} \leq B$. We are assuming further that for some $\bar{a}, m_{\bar{a}}=0$, while for another, $m_{\bar{a}}=B$.

Let $C$ be any integer such that $0<C<B$. Let

$$
S_{C}=\left\{\bar{a} \in \mathcal{V}(A) \backslash\{0\} \mid m_{\bar{a}}>C\right\} .
$$

Our claim is that the set $S_{C}$ is closed in the Zariski topology of $\hat{\mathcal{V}}(A)$. The demonstration that $S_{C}$ is closed is the main step and will occupy the next couple of paragraphs in the proof of the proposition.

Observe, that since $m_{\bar{a}}=0$ for some $\bar{a}$, we must have that $\operatorname{dim} M \equiv 1\left(\bmod p^{2}\right)$. This implies that for all $\bar{a}, m_{\bar{a}}$ is even. We also know that for $E \cong E_{\bar{a}}$, any $\bar{a}$, $\operatorname{dim} \Omega^{2 s}\left(\left.k\right|_{E}\right)=1+s p^{2}$ for any $s>0$. So let

$$
r=\left(\operatorname{dim} M-\operatorname{dim} \Omega^{2 c}(k)\right) / p^{2}
$$

where $c=C / 2$ if $C$ is even and $c=(C-1) / 2$ otherwise (so that in either case $2 c \leq C$ and $2 c+2>C)$. The important thing to notice is that for any $a$, the statement that $m_{\bar{a}}<C$ means that the dimension of the projective part of $M_{E_{\bar{a}}}$ is

$$
\operatorname{dim} M-\operatorname{dim} \Omega^{m_{\bar{a}}}(k) \geq p^{2} r .
$$

That is, if $m_{\bar{a}} \leq C$, then $M_{E_{\bar{a}}}$ has an $k E_{\bar{a}}$ free summand of rank at least $r$. In such a case, the rank of the matrix of the element $w_{\bar{a}}=v_{\bar{a}}^{p-1} u_{n}^{p-1}$ (which generates the socle of $\left.k E_{\bar{a}}\right)$ is at least $r$, by Lemma 2.1. On the other hand, by a similar dimension argument, if $m_{\bar{a}}>C$, then $M_{E_{\bar{a}}}$ has no $E_{\bar{a}}$-free summand of rank $r$, and the rank of the matrix of $w_{\bar{a}}$ is strictly less than $r$. So we have that $\bar{a} \in S_{C}$ if and only if the rank of $w_{\bar{a}}$ is strictly less than $r$.

Now we follow the argument of Theorem 3.5. Let $\mathcal{W}$ be the set of all $a=$ $\left(a_{1}, \ldots, a_{n-1}\right) \in k^{n-1}$ such that if $a \neq 0$, then $\bar{a}=\left[a_{1}, \ldots, a_{n-1}\right] \in \hat{\mathcal{V}}(A)$. Let $d=\operatorname{dim}(M)$ and let $\mathcal{S}$ be the set of all subsets of $\mathfrak{N}=\{1, \ldots, d\}$ having exactly $r$ elements. For any pair $S, T$ of elements of $\mathcal{S}$ let $f_{S, T}: \mathcal{W} \longrightarrow k$ be given by

$$
f_{S, T}(a)=\operatorname{Det}\left(M_{S, T}\left(w_{a}\right)\right)
$$

for $w_{a}=\left(a_{1} u_{1}+\cdots+a_{n-1} u_{n-1}\right)^{p-1} u_{n}^{p-1}$ and $a=\left(a_{1}, \ldots, a_{n-1}\right)$. Here, $M_{S, T}\left(w_{a}\right)$ is the $r \times r$ submatrix of the matrix of $w_{a}$ on the module $M$ having rows indexed by $S$ and columns indexed by $T$. It can be checked that the functions $f_{S, T}$ are homogeneous polynomial maps in $a_{1}, \ldots, a_{n-1}$. Hence, we have a polynomial map

$$
f=\Pi_{S, T \in \mathcal{S}} \quad f_{S, T}: \mathcal{W} \longrightarrow k^{\left(\begin{array}{l}
d \\
r
\end{array}\right)^{2}}
$$

with the property that $f(a)=0$ if and only if $\bar{a} \in S_{C}$. where $\bar{a}=\left[a_{1}, \ldots, a_{n-1}\right]$ is the corresponding element of $\hat{\mathcal{V}}(A)$. Because the zero locus of $f$ in $\mathcal{W}$ is a homogeneous closed set, we have that $S_{C}$ is closed in $\hat{\mathcal{V}}(A)$. 
Now we claim that for any such number $C, S_{C}$ is also open in $\hat{\mathcal{V}}(A)$. To see this fact, we replace $M$ by it dual $M^{*}$. Recall, that for any $t,\left(\Omega^{t}(k)\right)^{*} \cong \Omega^{-t}(k)$. For $M^{*}$ the values of $m_{\bar{a}}$ are all between $-B$ and 0 . Hence the next thing is to replace $M^{*}$ by $\Omega^{B}\left(M^{*}\right)$ so that the values of $m_{\bar{a}}$ for this module are all between 0 and $B$. But note that we have reversed the ordering. That is, for any $\bar{a}$, we have that

$$
\left.M\right|_{E_{\bar{a}}} \cong \Omega^{m_{\bar{a}}}(k) \oplus(\operatorname{proj}),
$$

and so

$$
\left.\left(\Omega^{B}\left(M^{*}\right)\right)\right|_{E_{\bar{a}}} \cong \Omega^{B-m_{\bar{a}}}(k) \oplus(\operatorname{proj}) .
$$

So that $S_{C}$ on $M$ becomes the complement of $S_{B-C}$ in $\hat{\mathcal{V}}(A)$. Hence, $S_{C}$ is both open and closed. Therefore, by the connectedness of $\hat{\mathcal{V}}(A)$ (Hypothesis 4.3) we have that $B=0$, and the proposition follows.

For the main result of this section we need a third hypothesis.

Hypothesis 4.5. Suppose that $G$ is a finite group scheme defined over $k$ and that the algebra $A=k G$ satisfies Hypotheses 4.1 and 4.3. We assume further that the ideal $A u_{n}=k G u_{n}$ generated by $u_{n}$ is a Hopf ideal so that $A / A u_{n}$ is a Hopf algebra and the group algebra of a finite group scheme. Moreover, we suppose that for any p-point $\alpha_{k}: k[t] /\left(t^{p}\right) \rightarrow A / A u_{n}$ one of the following two things happens.

(a) There exists a p-point $\beta: k[t] /\left(t^{p}\right) \rightarrow A$ such that the composition

$$
k[t] /\left(t^{p}\right) \longrightarrow k G \longrightarrow A / A u_{n}
$$

is a p-point equivalent to $\alpha$.

(b) There exist an element $x \in A$ such that $x+\left\langle u_{n}\right\rangle=\alpha(t) \in A / A u_{n}$, and we have that $x^{p}=y u_{n}$, where $y$ is a unit in $A$.

This last hypothesis seems somewhat unusual, but it is exactly what is needed. It allows us to prove the next theorem which is the main result of this section.

Theorem 4.6. Suppose that $A$ is an algebra which satisfies Hypotheses 4.1 , 4.3 and 4.5. Then the group of endotrivial modules for $A$ has the form $T(A) \cong \mathbb{Z}$ and is generated by the class of $\Omega(k)$.

Proof. Let $M$ be an endotrivial $A$-module, and suppose that the restriction $\left.M\right|_{E} \cong$ $\Omega^{m}\left(k_{E}\right) \oplus$ (proj) where $E$ is the subalgebra generated by $u_{n-1}$ and $u_{n}$. Our objective is to prove that $M \cong \Omega^{m}(k) \oplus$ (proj). This is equivalent to proving that $\Omega^{-m}(M) \cong k$, since $\Omega^{-m}(M)$ is indecomposable. Hence, for the proof we replace $M$ by $\Omega^{-m}(M)$ and we can assume that $\left.\left.M\right|_{E} \cong k\right|_{E} \oplus$ (proj).

Our first objective is to demonstrate that the module $\hat{M}=u_{n}^{p-1} M$ is a free module over $\hat{A}=A / A u_{n}$. For this purpose we use the method of $p$-points. Specifically, we show that if $\alpha: k[t] /\left(t^{p}\right) \rightarrow \hat{A}$ is a $p$-point, then the restriction $\alpha^{*}(\hat{M})$ is free module over $k[t] /\left(t^{p}\right)$. This is sufficient to prove that $\hat{M}$ is a projective $\hat{A}$-module.

Suppose that $\alpha$ is a $p$-point as above. We must consider the two situations of Hypothesis 4.5 separately. First suppose that we are in situation (a). In this case $\alpha$ is equivalent to $q \circ \beta$ where $\beta: k[t] /\left(t^{p}\right) \rightarrow A$ is a $p$-point and $q: A \rightarrow A / A u_{n}$ is the quotient map. By Hypothesis 4.3 we may assume that $v=\beta(t)=a_{1} u_{1}+\cdots+a_{n} u_{n}$ 
where $a_{i} \neq 0$ for some $i<n$. Let $E=\left\langle v, u_{n}\right\rangle$. By Theorem 4.4 we have that $\left.M\right|_{E} \cong k \oplus P$ where $P$ is a free $E$-module. As a consequence, $u_{n}^{p-1} M=u_{n}^{p-1} P$ is a free module on restriction to $k[t] /\left(t^{p}\right)$ along the map $\beta$. But then by the equivalences of $p$-points $\alpha^{*}(\hat{M})$ is also a free module.

Now consider the situation (b) in Hypothesis 4.5. Let $x \in A$ be as in the statement of (b), and let $H=\langle x\rangle$ be the subalgebra generated by $x$. Notice that $x^{p^{2}}=y^{p} u_{n}^{p}=$ 0 , while $x^{p^{2}-1}=y^{p-1} x^{p-1} u_{n}^{p-1} \neq 0$ since $x^{p-1}$ is not in the ideal generated by $u_{n}$ (see Hypothesis 4.1). Consequently, the subalgebra $H$ is isomorphic to $k[t] /\left(t^{p^{2}}\right)$, which is the group algebra of a cyclic group of order $p^{2}$ and hence is a finite group scheme. For any $p$-point $\gamma: k[t] /\left(t^{p}\right) \rightarrow H$ we must have that $\gamma(t)=z x^{p}=z y u_{n}$ where $z \in U$ is a unit. That is, there is only one equivalence class of $p$-points. However, we know that the restriction of $M$ to the subalgebra generated by $u_{n}$ is a trivial module plus a projective module. So the Jordan type of $\gamma(t)$ on $M$ must be the same as that of $u_{n}$ on $M$ which is $s[p]+[1]$ for some $s$. Hence, by Theorem 3.2 , the restriction to $H$ of $M$ is an endotrivial module. Because the dimension of $M$ is congruent to 1 modulo $p^{2}$ we know that $M \cong k \oplus P$ for some projective module $P$. That is, we recall that $\Omega^{2}\left(\left.k\right|_{H}\right) \cong k$ and that the dimension of $\Omega\left(\left.k\right|_{H}\right)$ is congruent to -1 modulo $p^{2}$. From this we get that $\alpha^{*}(\hat{M})$ is projective as in the previous case. As noted above, we have now shown that $\hat{M}$ is a free $\hat{A}$-module.

Now note that the socle of $\hat{A}$ is generated by the element $w=u_{1}^{p-1} \ldots u_{n-1}^{p-1}$. Hence the dimension of $\hat{M}$ is $p^{n-1} \operatorname{dim}(w \hat{M})$. Now $y=u_{n}^{p-1} w$ is a generator for the socle of $A$ and $w \hat{M}=y M$. Therefore, the module $M$ has a free submodule $Q$ of dimension $p^{n} \operatorname{dim}(y M)$. Because $p \operatorname{dim}(\hat{M})=\operatorname{dim}(M)-1$ we must have that $M \cong k \oplus Q$ by Lemma 2.1.

Remark 4.7. The importance of Hypothesis 4.3 is highlighted in the following example. Suppose that $U_{1}$ is the first infinitesimal Frobenius kernel of the unipotent subgroup of the algebraic group $G=S L_{3}$. That is, $k U_{1}$ is the restricted enveloping restricted $p$-Lie algebra of upper triangular $3 \times 3$ matrices. Then $k U_{1}$ satisfies Hypothesis 4.3 except in the case that $p=2$. It is generated by elements $u_{1}, u_{2}$ and $u_{3}$ which satisfy the equations

$$
u_{1}^{p}=0, u_{2}^{p}=0, u_{3}^{p}=0, \quad \text { and } \quad u_{3}=u_{1} u_{2}-u_{2} u_{1} .
$$

In the case that $p>2$, we have that $\left(a u_{1}+b u_{2}\right)^{p}=0$, hence Hypothesis 4.3 holds. However, if $p=2$, this is not the case. Indeed, if $p=2$ then the last equation can be replaced by $\left(u_{1} u_{2}\right)^{2}=\left(u_{2} u_{1}\right)^{2}$. These are the equations of the group algebra for a dihedral group. Consequently, $k U_{1}$ is isomorphic to the mod-2 group algebra of a dihedral group of order 8, at least as an algebra, though perhaps not as a Hopf algebra. Again we recall from [8] that the group of endotrivial module does not depend on the coalgebra structure. Therefore, $T\left(k U_{1}\right) \cong \mathbb{Z} \oplus \mathbb{Z}$ (see [14]).

\section{INFINITESIMAL UNIPOTENT SUBGROUPS OF ALGEBRAIC GROUPS}

The goal for the remainder of the paper is to investigate the properties of endotrivial modules for infinitesimal group schemes obtained by taking the Frobenius kernels of closed subgroup schemes of semisimple algebraic group schemes. In this 
section, we investigate some situations in which the unipotent subschemes satisfy the conditions of the last section.

Suppose that $G$ is a semisimple simply connected algebraic group defined and split over the finite field $\mathbb{F}_{p}$ with $p$ elements for a prime $p$. Here $k$ is the algebraic closure of $\mathbb{F}_{p}$. Let $\Phi$ be a root system associated to $G$ with respect to a maximal split torus $T$. Let $\Phi^{+}$(resp. $\Phi^{-}$) be the set of positive (resp. negative) roots and $\Delta$ be a base consisting of simple roots. Let $B$ be a Borel subgroup containing $T$ corresponding to the negative roots and let $U$ denote the unipotent radical of $B$.

If $H$ is an affine algebraic group scheme over $k$ and let $H_{r}=\operatorname{ker} F^{r}$. Here $F: H \rightarrow H^{(1)}$ is the Frobenius map and $F^{r}$ is the $r^{t h}$ iteration of the Frobenius map. We note that there is a categorical equivalence between restricted $\operatorname{Lie}(H)$-modules and $H_{1}$-modules. For each value of $r$, the group algebra $k H_{r}$ is the distribution algebra $\operatorname{Dist}\left(H_{r}\right)$ (see [26]). In general, for the rest of this paper, we use $\operatorname{Dist}\left(H_{r}\right)$ to denote the group algebra of $H_{r}$.

Theorem 5.1. Let $U$ be the unipotent radical of a Borel subgroup. For any $r \geq 1$, the algebra $\operatorname{Dist}\left(U_{r}\right)$ has a collection of elements $u_{1}, \ldots, u_{n}$ satisfying the conditions of Hypothesis 4.1. Here, $n=r\left|\Phi^{+}\right|$.

Proof. For any root $\alpha \in \Phi^{+}$, the distribution algebra of the infinitesimal root subgroup $\left(U_{\alpha}\right)_{r}$ is a divided powers algebra generated by elements

$$
v_{\alpha, i}=\frac{x_{\alpha}^{p^{i-1}}}{p^{i-1}} \quad \text { for } \quad i=1, \ldots, r .
$$

By convention we will set $v_{\alpha}=v_{\alpha, 1}$. Hence, we let $n=r\left|\Psi^{+}\right|$and let $u_{1}, \ldots, u_{n}$ be the elements $v_{\alpha, i}$ for $\alpha \in \Psi^{+}$and $i=1, \ldots, r$, ordered in such a way that for $u_{a}=v_{\alpha, i}$ and $u_{b}=v_{\beta, j}$, we have that $a<b$ if the height of the root $\alpha$ is less than the height of the root $\beta$. We know that for any positive roots $\alpha$ and $\beta$, the commutator $v_{\alpha, i} v_{\beta, j}-v_{\beta, j} v_{\alpha, i}$ is contained in the distribution algebra of the root subgroup $U_{\alpha+\beta}$ or is zero if $\alpha+\beta$ is not a root. In particular, that Condition (a) of Hypothesis 4.1 is satisfied is a standard calculation. Conditions (b) and (c) of Hypothesis 4.1 hold because the elements of collection $u_{1}, \ldots, u_{n}$ are ordered by increasing height of the roots. In particular, $u_{n}=v_{\alpha, j}$ where $\alpha$ has maximal height. Finally, Condition (d) is well known to be satisfied for $\operatorname{Dist}\left(U_{r}\right)$.

Our next goal is to determine when $T\left(U_{1}\right) \cong \mathbb{Z}$. Theorem 4.6 identifies the problem very clearly. We need to show that, except in the cases mentioned, Hypothesis 4.3 is satisfied. Throughout the proof, we assume the notation of the proof of Theorem 5.1. In particular, the elements $u_{1}, \ldots, u_{n}$ have the form $v_{\alpha, j}$ with the given ordering.

The first thing to notice is that the distribution algebra $\operatorname{Dist}\left(U_{1}\right)$ is isomorphic to the restricted enveloping algebra of the restricted $p$-Lie algebra $\mathfrak{u}$ of $U$. The restricted enveloping algebra is defined to be the quotient $\operatorname{Ten}(\mathfrak{u}) / J$ where $\operatorname{Ten}(\mathfrak{u})$ is the free tensor algebra

$$
\operatorname{Ten}(\mathfrak{u})=k \quad \oplus \quad \mathfrak{u} \quad \oplus \quad \mathfrak{u} \otimes \mathfrak{u} \quad \oplus \quad \mathfrak{u} \otimes \mathfrak{u} \otimes \mathfrak{u} \quad \oplus \quad \ldots
$$


and the ideal $J$ is generated by all elements of the form

$$
u \otimes v-v \otimes u-[u, v] \text { and } u^{\otimes p}-u^{[p]}
$$

for all $u, v \in \mathfrak{u}$. Here $u \rightarrow u^{[p]}$ is the $p$-power operation on the restricted Lie algebra $\mathfrak{u}$. Now by [25], we know that spectrum of $\mathrm{H}^{*}(u(\mathfrak{u}), k)$ is the restricted nullcone

$$
\mathcal{N}_{1}(\mathfrak{u})=\left\{u \in \mathfrak{u} \mid u^{[p]}=0\right\}
$$

where we are thinking of $\mathfrak{u}$ as a subspace of $\operatorname{Dist}\left(U_{1}\right)$. That is, every class of $p$-points is represented by a $p$-point having the form $\alpha: k[t] /\left(t^{p}\right) \longrightarrow \operatorname{Dist}\left(U_{1}\right)$, with $\alpha(t) \in \mathfrak{u}$. As a result, we may assume that $\mathcal{V}(A)=\mathcal{N}_{1}(\mathfrak{u}) \subset \mathfrak{u}$. The elements $u_{1}, \ldots, u_{n}$ can be taken to be a $k$-basis of $\mathfrak{u}$.

Notice that if the prime $p$ is larger than the Coxeter number, then $\hat{\mathcal{V}}(A)=k^{n-2}$ which is connected. Hence, we need only worry about small prime characteristics.

Proposition 5.2. If $p \geq 3$, then $A=\operatorname{Dist}\left(U_{1}\right)$ satisfies Hypothesis 4.3.

Proof. Suppose that $\bar{a}=\left[a_{1}, \ldots, a_{n}\right] \in \mathcal{V}(A)$ with $a_{i} \neq 0$ for some $i<n$. The point is that if $v=\sum a_{i} u_{i}$ and $p \geq 3$, then for any $a, b \in k$, not both zero, we have that $\left(a v+b u_{n-1}\right)^{p}=0$. That is, the choice of the ordering on the elements $u_{i}$ insures that $u_{n-1}$ has the form $v_{\alpha}$ for $\alpha \in \Phi^{+}$having next to greatest height. Because, $v^{p}=0$ and $p \geq 3$, we have that $\left(a v+b u_{n-1}\right)^{p}$ involves root subalgebras $\left(U_{\beta}\right)_{1}$, where $\beta$ has height at least two greater than $\alpha$. As there is no such $\beta$, we conclude that $\left(a v+b u_{n-1}\right)^{p}=0$, as asserted. Hence $\hat{\mathcal{V}}(A)$ is connected.

Proposition 5.3. If the algebraic group $G$ is semisimple but not simple, then $A=$ $\operatorname{Dist}\left(U_{1}\right)$ satisfies Hypothesis 4.3 .

Proof. We are assuming that $G$ is semisimple. If $G$ is not simple, then $G=G_{1} \times G_{2} \times$ $\cdots \times G_{t}$ and $A$ is a sum of algebras $A=A_{1} \oplus A_{2} \oplus \ldots A_{t}$. Each of the subalgebras has a nontrivial center. So there exist some $u_{i}$ for $i<n$ that is also central. In particular, we can assume that $u_{n-1}$ is also central. Consequently, if $v^{p}=0$, then so also is $\left(a v+b u_{n-1}\right)^{p}=0$ for any $(a, b) \in k^{2}$ with $a$ and $b$ not both zero. Thus, the points in $\hat{\mathcal{V}}(A)$ corresponding to $v$ and $u_{n-1}$ are in the same connected component of $\hat{\mathcal{V}}(A)$. Hence, there is only one connected component.

For the purposes of proving Theorem 5.6, we may assume that $r=1, p=2$ and that $G$ is a simple algebraic group. The case that $G$ has type $A_{2}$ was considered in the last section. Hypothesis 4.3 is vacuously satisfied (because $n=1$ ) if $G$ has type $A_{1}$. The remaining cases of groups of Lie rank 2 are settled in the following.

Proposition 5.4. Assume that $p=2$.

(1) If $G$ has type $B_{2}$, then $\mathcal{V}(A)$ has exactly two nonempty connected components and Hypothesis 4.3 is not satisfied by $A=\operatorname{Dist}\left(U_{1}\right)$.

(2) If $G$ has type $G_{2}$, then $A=\operatorname{Dist}\left(U_{1}\right)$ satisfies Hypothesis 4.3 .

Proof. In the first case, we may assume that $A=\operatorname{Dist}\left(U_{1}\right)$ is generated by elements $v_{\alpha}, v_{\beta}, v_{\alpha+\beta}$ and $v_{2 \alpha+\beta}$, where $\alpha$ and $\beta$ are the simple roots with $\beta$ long. Note that $v_{2 \alpha+\beta}$ is central (corresponding to $u_{n}$ in the previous notation). We may assume 
that $v_{\alpha} v_{\beta}+v_{\beta} v_{\alpha}=v_{\alpha+\beta}$ and that $v_{\alpha} v_{\alpha+\beta}+v_{\alpha+\beta} v_{\alpha}=v_{2 \alpha+\beta}$. Then expanding the equation

$$
\left(a v_{\alpha}+b v_{\beta}+c v_{\alpha+\beta}\right)^{2}=0
$$

we get that $a b=0$ and $a c=0$. Hence, $\hat{\mathcal{V}}(A)$ has two components which intersect in 0 .

The argument for $G$ of type $G_{2}$ is similar but with a different result. The distribution algebra is generated by,

$$
v_{\alpha}, v_{\beta}, v_{\alpha+\beta}, v_{2 \alpha+\beta}, v_{3 \alpha+\beta}, \text { and } v_{3 \alpha+2 \beta}
$$

where $\alpha$ and $\beta$ are the simple roots with $\beta$ long. This time, $v_{3 \alpha+2 \beta}$ is central. Expanding the equation

$$
\left(a v_{\alpha}+b v_{\beta}+c v_{\alpha+\beta}+d v_{2 \alpha+\beta}+e v_{3 \alpha+\beta}\right)^{2}=0
$$

we get that $a b=a c=a d=c d+b e=0$. If $b=c=d=0$, then there is no restriction on $e$. On the other hand, if $a=0$ then the component defined by $c d+b e=0$ is connected. Therefore, every $\bar{a}$ in $\hat{\mathcal{V}}(A)$ is in the connected component of $[0,0,0,0,1]$ which corresponds to $v_{3 \alpha+\beta}$.

Remark 5.5. It can be shown, as in the case of $A_{2}$ in the last section, that if $G$ has type $B_{2}$ and $p=2$, then $T\left(U_{1}\right) \cong \mathbb{Z} \oplus \mathbb{Z}$.

The following theorem describes the exact conditions when Hypothesis 4.3 is satisfied.

Theorem 5.6. Let $U$ be the unipotent radical of a Borel subgroup of the semisimple algebraic group $G$. Then $A=\operatorname{Dist}\left(U_{1}\right)$ satisfies Hypothesis 4.3 except in the cases that $p=2$, and $G$ has type either $A_{2}$ or $B_{2}$.

Proof. From Propositions 5.2, 5.3, 5.4 and Remark 5.5 stated above, we have reduced the proof of this theorem to dealing with the case that $p=2, r=1$ and $G$ is simple having Lie rank $\ell \geq 3$. The distribution algebra $A=\operatorname{Dist}\left(U_{1}\right)$ has generators $u_{1}, \ldots, u_{n}$ where for each $i, u_{i}=x_{\alpha}$ for $\alpha$ a positive root. That is, the elements $u_{i}$ can be indexed by the elements of the set $\Phi^{+}$of positive roots. Recall that the ordering on $u_{1}, \ldots, u_{n}$ respects the height of the corresponding roots. Thus $u_{n}$ corresponds to a root of maximal height.

In the arguments that follow we require some information about the three roots of greatest height in $\Phi^{+}$. This information is given in the following table. For each type of simple root systems and for each of the three roots of maximal height, the table gives the coefficients of the root expressed as a sum of simple roots. The ordering on the simple roots follows that of Bourbaki as given in [24]. This table was compiled from information given in [7] [24]. 


\begin{tabular}{|c|c|c|c|}
\hline Type & $\beta_{1}$ & $\beta_{2}$ & $\beta_{3}$ \\
\hline$A_{l}$ & $(1,1, \ldots, 1,0)$ & $(0,1,1, \ldots, 1)$ & $(1,1, \ldots, 1)$ \\
\hline$B_{l}$ & $(0,1,2, \ldots, 2)$ & $(1,1,2, \ldots, 2)$ & $(1,2,2, \ldots, 2)$ \\
\hline$C_{l}$ & $(1,1,2, \ldots, 2,1)$ & $(1,2, \ldots, 2,1)$ & $(2,2, \ldots, 2,1)$ \\
\hline$D_{l}$ & $(0,1,2, \ldots, 2,1,1)$ & $(1,1,2, \ldots, 2,1,1)$ & $(1,2, \ldots, 2,1,1)$ \\
\hline$E_{6}$ & $(1,1,2,2,2,1)$ & $(1,1,2,3,2,1)$ & $(1,2,2,3,2,1)$ \\
\hline$E_{7}$ & $(1,2,2,4,3,2,1)$ & $(1,2,3,4,3,2,1)$ & $(2,2,3,4,3,2,1)$ \\
\hline$E_{8}$ & $(1,2,4,6,5,4,3,2)$ & $(1,3,4,6,5,4,3,2)$ & $(2,3,4,6,5,4,3,2)$ \\
\hline$F_{4}$ & $(1,2,4,2)$ & $(1,3,4,2)$ & $(2,3,4,2)$ \\
\hline
\end{tabular}

The first case that we consider is the one in which the root system of $G$ has two roots with (the same) next to maximal height. This is the case, for example, if $G$ has type $A_{n}$. In this case, $u_{n-2}$ and $u_{n-1}$ correspond to roots $\beta_{1}$ and $\beta_{2}$ having the same height (see the table). Because the Lie rank of $G$ is larger than 2, we must have that any element of the form $v=a u_{n-2}+b u_{n-1}$ has $v^{2}=0$ and hence $[0, \ldots, a, b] \in \hat{\mathcal{V}}(A)$. As a consequence, all such elements are in the same connected component (call it $\mathcal{C}$ ) of $\hat{\mathcal{V}}(A)$. Suppose that $\bar{a}=\left[a_{1}, \ldots, a_{n-1}\right]$ is in $\hat{\mathcal{V}}(A)$. Let $v=a_{1} u_{1}+\cdots+a_{n-1} u_{n-1}$. Then the commutators $\left[v, u_{n-2}\right]=v u_{n-2}-u_{n-2} v$ and $\left[v, u_{n-1}\right]$ are both elements of the space generated by $u_{n}$, because of the rank considerations. Consequently, there is some combination $w=c u_{n-2}+d u_{n-1}$ such that $[v, w]=0$. Hence, we see that $\bar{a}$ is in the connected component $\mathcal{C}$ that contains the point corresponding to $w$.

The other possible case is that $u_{n-2}$ and $u_{n-1}$ correspond to roots of different height. That is, we are assuming that there is only one root of next to maximal height. Let $\Delta=\left\{\alpha_{1}, \ldots, \alpha_{\ell}\right\}$ be the simple roots. There is no loss of generality in assuming here that $u_{i}=x_{\alpha_{i}}$ for $i=1, \ldots, \ell$. Let $\beta_{1}, \beta_{2}$ and $\beta_{3}$ be the roots corresponding to the elements $u_{n-2}, u_{n-1}$ and $u_{n}$, respectively (see the table). Our assumption on heights requires that there be some simple root $\alpha_{i}$ and another simple root $\alpha_{j}$ such that $\beta_{2}=\beta_{1}+\alpha_{i}$ and $\beta_{3}=\beta_{2}+\alpha_{j}$. In these circumstances, we know that $\alpha_{i}+\alpha_{j}$ is a root of height 2 .

As in the previous case, because $\ell \geq 3$ we know that $\beta_{1}$ must have height at least 2 , and so $u_{n-2}$ and $u_{n-1}$ are in the same connected component $\mathcal{C}$ of $\hat{\mathcal{V}}(A)$. That is, the commutator $\left[u_{n-2}, u_{n-1}\right]=0$. Moreover any linear combination of $u_{n-2}$ and $u_{n-1}$ is also in $\mathcal{C}$. Choose any element $v=a_{1} u_{1}+\cdots+a_{n-1} u_{n-1}$ with $v^{2}=0$. We must have that $a_{i} a_{j}=0$ ( $i$ and $j$ as above), as otherwise $v^{2}$ would have a nonzero coefficient on the basis element $u_{s}$ corresponding to the root $\alpha_{i}+\alpha_{j}$. So either $a_{i}=0$ or $a_{j}=0$. In the second case $\left(a_{j}=0\right)$, the commutator $\left[v, u_{n-1}\right]=0$.

So consider the first case, that $a_{i}=0$. then we must have that $\left[v, u_{n-2}\right]=c a_{s} u_{n}$ for some constant $c$ where $u_{s}$ corresponds to the root $\alpha_{i}+\alpha_{j}$. At the same time $\left[v, u_{n-1}\right]=d a_{j} u_{n}$. for some constant $d$. The numbers $c$ and $d$ only depend on the choice of the basis $u_{1}, \ldots, u_{n}$ and not on the choice of $v$. So in any event, some linear combination $w=a u_{n-2}+b u_{n-1}$ has the property that $[v, w]=0$. Hence, the point of $\hat{\mathcal{V}}(A)$ corresponding to $v$ is in the same connected component as that of $w$ and that component is $\mathcal{C}$. 
We can now determine the group of endotrivial modules for $U_{r}$, in the case that $r=1$.

Theorem 5.7. Let $U$ be the unipotent radical of a Borel subgroup of the semisimple algebraic group $G$. Then $T\left(U_{1}\right) \cong \mathbb{Z}$ and is generated by the class or $\Omega(k)$ except in the cases that $\Phi=A_{1}$ or that $p=2$ and $\Phi=A_{2}$ or $B_{2}$.

Proof. Let $\mathfrak{u}$ be the restricted $p$-Lie algebra of $U$. We have shown that $\operatorname{Dist}\left(U_{1}\right)$ satisfies Hypothesis 4.1 and in the proof we see that the elements $u_{1}, \ldots, u_{n}$ satisfying the hypothesis can be taken to be a basis of $\mathfrak{u} \subseteq \operatorname{Dist}\left(U_{1}\right)$.

All of the above applies to the group scheme $\hat{U}_{1}$ whose distribution algebra is $\operatorname{Dist}\left(U_{1}\right) / \operatorname{Dist}\left(U_{1}\right) u_{n}$. This is the restricted enveloping algebra of the restricted $p$-Lie algebra $\hat{\mathfrak{u}} /\left\langle u_{n}\right\rangle$. Here $\left\langle u_{n}\right\rangle$ denotes the Lie subalgebra generated by $u_{n}$ and having dimension one. Any equivalence class of $p$-points for $\operatorname{Dist}\left(\hat{U}_{1}\right)$ is represented by one of the form $\alpha: k[t] /\left(t^{p}\right) \longrightarrow \operatorname{Dist}\left(\hat{U}_{1}\right)$ such that $\alpha(t) \in \hat{\mathfrak{u}}$. Let $x \in \mathfrak{u}$ be an element such that $x+\left\langle u_{n}\right\rangle=\alpha(t)$. We know that as an elements of $\operatorname{Dist}\left(U_{1}\right)$, $x^{p}=x^{[p]} \in\left\langle u_{n}\right\rangle$ since $\alpha(t)^{p}=0$ in $\operatorname{Dist}\left(\hat{U}_{1}\right)$. It follows that either $x^{p}=0$ or $x^{p}$ is a multiple (by an element of $k$ ) of $u_{n}$. In the former case the $p$-point $\alpha$ lifts to the $p$-point $\beta: k[t] /\left(t^{p}\right) \longrightarrow \operatorname{Dist}\left(U_{1}\right)$, given by $\beta(t)=x$.

Therefore, we have shown that $U_{1}$ satisfies Hypothesis 4.5. Hence, the theorem follows from Theorem 4.6.

\section{ENDOtRIVIAL MODULES OVER PARABOLIC SUBGROUPS}

In this section we show that we can use the results of the last section to obtain information on infinitesimal subgroups of parabolic subgroups. We maintain the notation of the last section and the same assumptions on the group $G$. First we consider the case of the Borel subgroup.

For any group scheme $H$, let $\bmod (H)$ be the category of finite dimensional rational $H$-modules. This construction can be applied when $H=G, B, P_{J}, L_{J}, U, U_{J}$ and $T$. Let $X\left(T_{r}\right)$ be the set of characters of $T_{r}$ which can be indentified with the set of one dimensional simple modules for $T_{r}$.

Theorem 6.1. Let $U$ be the unipotent radical of $B$. Then we have the following.

(a) Suppose that $\operatorname{dim} U=1$. Then $T\left(U_{1}\right) \cong\{1\}$ if $p=2, T\left(U_{1}\right) \cong \mathbb{Z} / 2 \mathbb{Z}$ if $p>2$ and $T\left(U_{r}\right) \cong \mathbb{Z}$ for $r \geq 2$.

(b) In any case, $T\left(B_{1}\right) \cong X\left(T_{1}\right) \otimes T\left(U_{1}\right)$

Proof. To prove part (a) note that the $\operatorname{Dist}\left(U_{r}\right)$ is isomorphic to the group algebra of an elementary abelian $p$-subgroup $E$ of rank $r$. Consequently, $T\left(U_{r}\right)=T(E)$ and is as given in the theorem.

For part (b), suppose that $M$ is an indecomposable endotrivial module for $B_{1}$. By the results of the last section, the image of the restriction of $T\left(B_{1}\right)$ to $T\left(U_{1}\right)$ is generated by the class of $\Omega\left(\left.k\right|_{U_{1}}\right)$ and by at most one other class. It can be shown, using methods similar to those in [12, 13] that the restriction map is surjective. In the case that $T\left(U_{1}\right) \cong \mathbb{Z}$ is generated by the class of $\Omega(k)$, this is obvious. For the cases that $p=2$ and $G$ is of type $A_{2}$, or $B_{2}$ we must rely on the fact that the 
cohomology $\mathrm{H}^{*}\left(B_{1}, k\right)$ is finitely generated over the subalgebra generated by degree 2 elements (cf. [20, Section 1]). In these siituations we may choose inverse images for the generators of $T\left(U_{1}\right)$ under the restriction map. One of these can be taken to be $\Omega(k)$. The other is constructed using the cohomological push-out method used in $[12,13]$.

Now recall that an indecomposable module over $B_{1}$ must be indecomposable when restricted to $U_{1}$. As a result, if $N$ is a suitable product of the inverse images of the generators such that $N_{U_{1}}$ is in the same class as $M_{U_{1}}$ modulo projectives, then $M \otimes N^{*}$ is in the class of $k$ on restriction to $U_{1}$ and its class contains a one dimensional module. In particular, it is an element of $X\left(T_{1}\right)$ as asserted.

For notation in what follows, let $J \subseteq \Delta$, with $U_{J}$ the unipotent radical of parabolic subgroup $P_{J}$. The Levi subgroup is denoted by $L_{J}$ with $P_{J}=L_{J} \ltimes U_{J}$.

In what follows we assume that $T\left(U_{r}\right) \cong \mathbb{Z}$. If $r=1$, then this is equivalent to the assumption that we are not in one of the exceptional cases of Theorem 5.6. The exceptional cases can be handled similarly, but with more work.

For parabolic subgroups $P_{J}$ where $J$ is a nonempty proper subset of $\Delta$, the only endotrivial modules for $\left(P_{J}\right)_{r}$ arise from syzygies of the trivial module.

Theorem 6.2. Assume that $\operatorname{dim} U \geq 2$ and $T\left(U_{r}\right)$ is generated by $\Omega(k)$. Let $J$ be a proper subset of $\Delta$. Then $T\left(\left(P_{J}\right)_{r}\right)=X\left(\left(P_{J}\right)_{r}\right) \otimes \mathbb{Z}$.

Proof. Let $M$ be an indecomposable endotrivial module over $\left(P_{J}\right)_{r}$. Then $M$ is endotrivial over $U_{r}$. Since $\operatorname{dim} U \geq 2$, it follows that $\left.M\right|_{U_{r}} \cong \Omega^{n}(k) \oplus$ (proj) so we can assume using dimension shifting that $\left.M\right|_{U_{r}} \cong k \oplus(\operatorname{proj})$. Hence $\left.M\right|_{\left(U_{J}\right)_{r}} \cong$ $k \oplus$ (proj). Now let $U^{\prime}$ be another unipotent radical for some other Borel subgroup $B \subset P_{J}$. Then $\left.M\right|_{U_{r}^{\prime}} \cong \Omega^{t}(k) \oplus$ (proj) for some $t$. However, $U_{J} \subseteq U^{\prime} \cap U$ so $\left.M\right|_{\left(U_{J}\right)_{r}} \cong \Omega^{t}(k) \oplus$ (proj). Thus, $t=0$ and $\left.M\right|_{U_{r}^{\prime}} \cong k \oplus$ (proj).

We also note that the condition that $\operatorname{dim} U \geq 2$ insures that the rank of $\Phi$ is greater than two, thus $\operatorname{dim} U_{J} \geq 2$ for $J$ a proper subset of $\Delta$. Moreover, one can verify that $\operatorname{dim} \mathcal{N}\left(\mathfrak{u}_{J}\right) \geq 2$. This insures that $\left(U_{J}\right)_{1}$ has nonperiodic cohomology, so that $\Omega^{t}(k) \neq k$ unless $t=0$.

Next we observe that $U_{J}$ is contained in every maximal unipotent subgroup of $P_{J}$. Let $H$ be a maximal unipotent subgroup and take $H U_{J}$. Since $U_{J}$ is a normal subgroup of $P_{J}, H U_{J}$ is a subalgebra and unipotent. Thus by maximality, $U_{J} \leq H$. Moreover, one has $H=\hat{U} \ltimes U_{J}$ where $\hat{U}$ is a maximal unipotent subgroup in $L_{J}$.

Let $L=M^{\left(U_{J}\right)_{r}}$ be the fixed points of $M$ under $\left(U_{J}\right)_{r}$. We claim that $L$ is an endotrivial $\left(L_{J}\right)_{r}$-module. Any $\pi$-point, $\alpha: k[t] /\left(t^{p}\right) \rightarrow \operatorname{Dist}\left(\left(L_{J}\right)_{r}\right)$ factors as $\alpha: k[t] /\left(t^{p}\right) \rightarrow \operatorname{Dist}\left(\left(U^{\prime}\right)_{r}\right) \rightarrow \operatorname{Dist}\left(\left(U^{\prime}\right)_{r} / U_{r}\right) \rightarrow \operatorname{Dist}\left(\left(L_{J}\right)_{r}\right)$ for some unipotent radical $U^{\prime}$ of a Borel subgroup in $P_{J}$. We have $\left.M\right|_{U_{r}^{\prime}} \cong k \oplus(\operatorname{proj})$ so

$$
\left.L\right|_{U_{r}^{\prime}}=\left.M^{\left(U_{J}\right)_{r}}\right|_{U_{r}^{\prime}} \cong k \oplus(\operatorname{proj})^{\left(U_{J}\right)_{r}} .
$$

Note that $(\operatorname{proj})^{\left(U_{J}\right)_{r}}$ is a projective $U_{r}^{\prime} / U_{r}$-module. The restriction of $M$ along $\alpha$ to a $\left(k[t] /\left(t^{p}\right)\right)$-module decomposes as $k \oplus$ (proj). So the fixed point module $M^{\left(U_{J}\right)_{r}}$ has constant Jordan type [1] + (proj). Consequently, $M^{\left(U_{J}\right)_{r}}$ is endotrivial as an $\left(L_{J}\right)_{r}$-module by Theorem 3.2 . 
If $\bar{u}$ be a generator for the socle of $\operatorname{Dist}\left(\left(U_{J}\right)_{r}\right)$, then

$$
\bar{u} M \subseteq M^{\left(U_{J}\right)_{r}}
$$

and there is a short exact sequence of $\left(P_{J}\right)_{r}$-modules

$$
0 \rightarrow \bar{u} M \rightarrow M^{\left(U_{J}\right)_{r}} \rightarrow \lambda \rightarrow 0
$$

where $\lambda \in X\left(\left(P_{J}\right)_{r}\right)$. By the preceding argument, $\bar{u} M$ is a free $\operatorname{Dist}\left(\left(U^{\prime}\right)_{1} /\left(U_{J}\right)_{r}\right)$, so $\bar{u} M$ has constant Jordan type. Consequently, $\bar{u} M$ is a projective $\left(L_{J}\right)_{r}$-module because $\bar{u}$ is normalized by elements in $\left(P_{J}\right)_{r}$.

It follows that, as an $\left(L_{J}\right)_{r}$-module, $M^{\left(U_{J}\right)_{r}} \cong \lambda \oplus(\operatorname{proj})$. Let $\hat{\imath}: \lambda \rightarrow M^{\left(U_{J}\right)_{r}}$. We can compose this with the inclusion of $M^{\left(U_{J}\right)_{r}} \subseteq M$ to obtain a $\left(P_{J}\right)_{r}$-homomorphism: $\iota: \lambda \rightarrow M$. We obtain a short exact sequence,

$$
0 \rightarrow \lambda \stackrel{\iota}{\rightarrow} M \rightarrow Q \rightarrow 0
$$

of $\left(P_{J}\right)_{r}$-modules.

Note that $\iota(\lambda) \nsubseteq \bar{u} M$ and $\left.M\right|_{U_{r}^{\prime}} \cong \lambda \oplus R$ where $R$ is projective. So all $U_{r}^{\prime}$-fixed points of $R$ are in $\bar{u} M=\bar{u} R$. It follows that $\iota(\lambda) \nsubseteq R$. Hence, $Q \cong M / \iota(\lambda)$ is a projective $U_{r}^{\prime}$-module because

$$
\left.Q\right|_{U_{r}^{\prime}} \cong(R \oplus k) / \iota(\lambda) \cong R
$$

Any $\pi$-point factors through some $\left(U^{\prime}\right)_{r}$ thus $Q$ is projective as a module over $\left(P_{J}\right)_{r}$. Since $M$ is indecomposable we must have $M \cong \lambda$.

\section{Restriction of Weyl modules over $S L_{2}$ to Unipotent Subschemes}

In this section we adhere to the notation presented in Section 5. In addition, let the Euclidean space associated with the root system $\Phi$ be denoted by $\mathbb{E}$ and the inner product on $\mathbb{E}$ by $\langle$,$\rangle . The Weyl group W$ is the group generated by reflections associated to the root system $\Phi$ and the affine Weyl group $W_{p}$ is the group generated by $W$ and translations by elements in $p \mathbb{Z} \Phi$. Let $X(T)$ be the integral weight lattice obtained from $\Phi$. The set $X(T)$ has a partial ordering defined as follows. If $\lambda, \mu \in X(T)$, then $\lambda \geq \mu$ if and only if $\lambda-\mu \in \sum_{\alpha \in \Pi} \mathbb{N} \alpha$. If $\alpha^{\vee}=2 \alpha /\langle\alpha, \alpha\rangle$ is the coroot corresponding to $\alpha \in \Phi$, then the set of dominant integral weights is defined by

$$
X(T)_{+}=\left\{\lambda \in X(T): 0 \leq\left\langle\lambda, \alpha^{\vee}\right\rangle \text { for all } \alpha \in \Delta\right\} .
$$

Furthermore, the set of $p^{r}$-restricted weights is

$$
X_{r}(T)=\left\{\lambda \in X(T): 0 \leq\left\langle\lambda, \alpha^{\vee}\right\rangle<p^{r} \text { for all } \alpha \in \Delta\right\} .
$$

The affine Weyl group $W_{p}$ acts on $X(T)$ via the "dot action" given by $w \cdot \lambda=$ $w(\lambda+\rho)-\rho$ where $w \in W_{p}, \lambda \in X(T)$, and $\rho$ is the half sum of positive roots.

For a reductive algebraic group $G$, the simple modules will be denoted by $L(\lambda)$ and the induced modules by $H^{0}(\lambda)=\operatorname{ind}_{B}^{G} \lambda$, where $\lambda \in X(T)_{+}$. The Weyl module $V(\lambda)$ has simple head $L(\lambda)$ and is defined as $V(\lambda)=H^{0}\left(-w_{0} \lambda\right)^{*}$. For the infinitesimal group scheme $G_{r}$, the simple modules will be denoted by $L_{r}(\lambda)$. If $\lambda \in X_{r}(T)$, then $L_{r}(\lambda) \cong L(\lambda)$ as modules over $G_{r}$. Also, for $\lambda \in X(T)_{+}$, let $T(\lambda)$ be the indecomposable tilting module with highest weight $\lambda$. 
For the rest of this section, suppose that $G=S L_{2}$. Let $U$ be the unipotent radical of $B$ (negative Borel subgroup). Note $X(T)_{+}=\mathbb{N}$ and $\operatorname{dim} U=1$. The algebra $k U_{r}=\operatorname{Dist}\left(U_{r}\right)$ is a divided power algebra with basis $\left\{x^{i} / i ! \mid i=0,1, \ldots, p^{r}-1\right\}$ with relations

$$
\frac{x^{i}}{i !} \cdot \frac{x^{j}}{j !}=\left(\begin{array}{c}
i+j \\
i
\end{array}\right) \frac{x^{i+j}}{(i+j) !}
$$

for all $i$ and $j$ such that $i+j<p^{r}$. The product is zero of $i+j \geq p^{r}$.

Let $W$ be the natural 2 dimensional module for $G$. It has a basis $\left\{v_{1}, v_{2}\right\}$ such that $x v_{1}=v_{2}$ and $x v_{2}=0$. The Weyl module $V(\lambda)$ is the $\lambda^{t h}$ symmetric product $S^{\lambda}(W)$ (viewed as a quotient of $W^{\otimes \lambda}$ ). It has dimension $\lambda+1$. The highest weight element in $V(\lambda)$ is the element $w_{0}=v_{1} \otimes \cdots \otimes v_{1}$, with $\lambda$ factors in the tensor product. The module $V(\lambda)$ has a basis consisting of $w_{0}$ and all $w_{i}$, as given below, for $i=2, \ldots, \lambda$. The element $w_{i}$ is the sum in $S^{\lambda}(W)$ of all monomials of the form $v_{s(1)} \otimes v_{s(2)} \otimes \cdots \otimes v_{s(\lambda)}$ where $s$ runs through the collection of all function $s:\{1, \ldots, \lambda\} \rightarrow\{1,2\}$ such that $i$ value of $s$ are equal to 2 and $\lambda-i$ values of $s$ are equal to 1 . Clearly there are $\left(\begin{array}{c}\lambda \\ i\end{array}\right)$ such monomials.

Recall that if $L$ and $M$ are $k U$-modules, then the action of $k U$ on $M \otimes N$ is given by

$$
\frac{x^{t}}{t !}(\ell \otimes m)=\sum_{j=0}^{t} \frac{x^{j}}{j !} \ell \otimes \frac{x^{t-j}}{(t-j) !} m
$$

for all $\ell \in L, m \in M$ and all $t \leq p^{r}-1$. The aforementioned action is imposed by the Hopf algebra structure on $k U$. The first result that we need is the following.

Lemma 7.1. Let $j$ and $t$ be nonnegative integers such that $j+t \leq \lambda$. Then

$$
\frac{x^{j}}{j !} w_{t}=\left(\begin{array}{c}
t+j \\
j
\end{array}\right) w_{t+j}
$$

If $t+j>\lambda$, then $\left(x^{j} /(j !)\right) w_{t}=0$.

Proof. We notice first that $\left(x^{i} /(i !)\right) v_{j}=0$ for $i \geq 2$. As a consequence, we have that $\left(x^{i} /(i !)\right) w_{0}=w_{i}$ provided $i<\lambda$ and $i<p^{r}$. This observation is enough to prove the lemma in the case that $t+j<p^{r}$. To verify the general case we must count monomials. Hence, one way to proceed would be to embed $U=U_{r}$ in $U_{s}$ for $s$ sufficiently large. On the other hand, it is a simple task to count monomial terms.

Suppose that $u=u_{1} \otimes \cdots \otimes u_{\lambda}$ is a monomial such that exactly $t$ of the $u_{i}^{\prime} s$ are equal to $v_{2}$ and the rest equal to $v_{1}$. Then the expression of $\left(x^{j} /(j !)\right) u$ as a sum of monomial terms, has $\left(\begin{array}{c}\lambda-t \\ j\end{array}\right)$ such terms. Moreover, all such terms have exactly $t+j$ factors equal to $v_{2}$ while the rest are equal to $v_{1}$. That is, $x^{j} /(j !) \cdot w_{t}$ is a multiple of $w_{t+j}$. Now, $w_{t+j}$ has exactly $\left(\begin{array}{c}\lambda \\ t+j\end{array}\right)$ total terms. Because there are $\left(\begin{array}{l}\lambda \\ t\end{array}\right)$ such $u$ in the expression of $w_{t}$, we must have that the coefficient on each term in the expression of $\left(x^{j} /(j !)\right) w_{t}$ is

$$
\frac{\left(\begin{array}{c}
\lambda-t \\
j
\end{array}\right)\left(\begin{array}{c}
\lambda \\
t+j
\end{array}\right)}{\left(\begin{array}{c}
\lambda \\
t
\end{array}\right)}=\frac{(\lambda-t) !}{j !(\lambda-t-j) !} \frac{\lambda !}{(t) !(\lambda-t) !} \frac{(t+j) !(\lambda-t-j) !}{\lambda !}=\left(\begin{array}{c}
t+j \\
j
\end{array}\right)
$$

as asserted. 
With this formula we can prove the following result.

Proposition 7.2. Let $n$ be any positive integer. Then the restriction of $V\left(n p^{r}\right)$ to $U_{r}$ is the direct sum

$$
\left.V\left(n p^{r}\right)\right|_{U_{r}} \cong k \oplus\left(k U_{r}\right)^{\oplus n}
$$

Proof. Recall that $k U_{r}$ is a local ring with a simple socle that is spanned as a $k$-vector space by the element $x^{p^{r}-1} /\left(p^{r}-1\right)$ !. In particular, it is a self injective algebra, and hence projective $k U_{r}$-modules are both free and injective.

Suppose that $Q$ is free $k U_{r}$-module with $k U_{r}$-generators $q_{1}, \ldots, q_{n}$. Define a homomorphism $\psi: Q \longrightarrow V\left(n p^{r}\right)$ by the assignment $\psi\left(q_{i}\right)=w_{(i-1)\left(p^{r}\right)}$. Thus we have that

$$
\psi\left(\frac{x^{p^{r}-1}}{\left(p^{r}-1\right) !} q_{i}\right)=\left(\begin{array}{c}
(i-1) p^{r}+p^{r}-1 \\
p^{r}-1
\end{array}\right) w_{i p^{r}-1} \neq 0
$$

The reason why the above expression is not zero is that

$$
\left(\begin{array}{c}
(i-1) p^{r}+p^{r}-1 \\
p^{r}-1
\end{array}\right)=\prod_{j=0}^{p^{r}-2} \frac{i p^{r}-1-j}{p^{r}-1-j}
$$

where in each factor the same power of $p$ divides the numerator as divides the denominator. That is, for $0 \leq j \leq p^{r}-2$, the highest power of $p$ which divides either $i p^{r}-1-j$ or $p^{r}-1-j$ is the same as the highest power of $p$ which divides $1+j$. This proves that the map $\psi$ is injective since it is injective on the socle of $Q$. Moreover, the image of $\psi$ has codimension one in $W$. Hence we have an exact sequence

$$
0 \longrightarrow Q \stackrel{\psi}{\longrightarrow} W \longrightarrow k \longrightarrow 0
$$

which is split because $Q$ is an injective module.

Theorem 7.3. Let $G=S L_{2}$. The Weyl module $V(\lambda)$ (resp. $H^{0}(\lambda)$ ) is endotrivial over $G_{r}$ if and only if $\lambda=n p^{r}$ or $n p^{r}-2$ for $n \geq 0$.

Proof. First observe that $\operatorname{dim} V(\lambda)=\lambda+1$. If $\left.V(\lambda)\right|_{G_{r}}$ is endotrivial then $\operatorname{dim} V(\lambda) \equiv$ $\pm 1 \bmod p^{r}$. This implies that $\lambda=n p^{r}$ or $n p^{r}-2$ where $n \geq 0$.

Next we know that $V\left(n p^{r}\right)$ is endotrivial as a $U_{r}$-module. Because $V\left(n p^{r}\right)$ is a $G$-module, $V\left(n p^{r}\right)$ is an endotrivial module for any maximal unipotent subgroup scheme $U_{r}^{\prime}$ of $G_{r}$. Now every $\pi$-point factors through one of these group schemes. Consequently, the result follows from Theorem 3.2 .

Let $T_{\lambda}^{\mu}$ be the translation functor as given in [26, Chapter 7]. According to [26, II. 7.19 Proposition], there exists a short exact sequence of $G$-modules

$$
0 \rightarrow V\left(n p^{r}\right) \rightarrow T_{-1}^{0}\left(V\left(n p^{r}-1\right)\right) \rightarrow V\left(n p^{r}-2\right) \rightarrow 0 .
$$

But, $\left.T_{-1}^{0}\left(V\left(n p^{r}-1\right)\right)\right|_{G_{r}}$ is a projective (also injective) $G_{r}$-module, thus an injective $U_{r}$-module. It follows that $\left.V\left(n p^{r}-2\right)\right|_{U_{r}} \cong \Omega^{-1}(k) \oplus(\operatorname{proj})$ and $\left.V\left(n p^{r}-2\right)\right|_{U_{r}}$ is endotrivial over $U_{r}$. The argument in the preceding paragraph can be used to conclude that $\left.V\left(n p^{r}-2\right)\right|_{G_{r}}$ is endotrivial.

Corollary 7.4. Let $G=S L_{2}$. 
(a) The simple module $L(\lambda)$ is endotrivial over $G_{r}$ if and only if $r=1$ and $\lambda=0, p-2$.

(b) The tilting module $\left.T(\lambda)\right|_{G_{r}}$ is endotrivial if and only if $r=1$ and $\lambda=0, p-2$.

Proof. (a) Let $\lambda \in X(T)_{+}$and assume that $L(\lambda)$ is endotrivial over $G_{r}$. Then $\left.L(\lambda)\right|_{G_{1}}$ must be a direct sum of an endotrivial $G_{1}$-module with a projective $G_{1^{-}}$ module. By Steinberg's Tensor Product Theorem, $L(\lambda)=L\left(\lambda_{0}\right) \otimes L(\mu)^{(1)}$ where $\lambda \in X_{1}(T)$ and $\mu \in X(T)_{+}$. Therefore, $\left.L(\lambda)\right|_{G_{1}} \cong L\left(\lambda_{0}\right)^{\oplus n}$ where $n=\operatorname{dim} L(\mu)^{(1)}$. Combining this with the fact mentioned above means that $\mu=0$ and $L(\lambda)=L\left(\lambda_{0}\right)$, thus $\lambda \in X_{1}(T)$.

Now when $\Phi$ is of type $A_{1}, V(\lambda)=L(\lambda)$ for all $\lambda \in X_{1}(T)$. Thus the result follows by using Theorem 7.3 .

(b) Suppose that $\left.T(\lambda)\right|_{G_{r}}$ is endotrivial. If $\lambda \geq p-1$ then $\left.T(\lambda)\right|_{G_{1}}$ is projective [26, E.8. Lemma], and $p \mid \operatorname{dim} T(\lambda)$. Thus we can assume that $0 \leq \lambda \leq p-2$. The only possibilities for endotrivial modules for $\left.T(\lambda)\right|_{G_{r}}$ occur when $\lambda=0, p-2$ when $r=1$ because in these cases $T(\lambda) \cong V(\lambda)$ for $0 \leq \lambda \leq p-1$.

Finally, we can determine the structure of the endotrivial group for $G_{1}=\left(S L_{2}\right)_{1}$.

Corollary 7.5. Let $G=S L_{2}$. Then $T\left(G_{1}\right) \cong \mathbb{Z} \oplus \mathbb{Z} / 2 \mathbb{Z}$.

Proof. The category of $G_{1}=\left(S L_{2}\right)_{1}$-modules has tame representation type and the indecomposable module are classified (cf. [11, Section 3]). Using this classification, one see that

$$
T\left(G_{1}\right)=\left\{\Omega^{m}(k), \Omega^{n}(L(p-2)): m, n \in \mathbb{Z}\right\} .
$$

Moreover, $L(p-2) \cong L(p-2)^{*}$, thus $T\left(G_{1}\right)=\left\langle\Omega^{1}(k), L(p-2)\right\rangle \cong \mathbb{Z} \oplus \mathbb{Z} / 2 \mathbb{Z}$.

\section{Rational Endotrivial Modules over $G$}

Our aim is to investigate the question of when a rational $G$-module is an endotrivial module over any of the infinitesimal subgroups of $G$. We show that if the Lie rank of $G$ is at least 2 then the (nontrivial) irreducible modules for $G_{r}$ as well as the (nontrivial) Weyl modules and tilting modules are not endotrivial. The situation is strikingly different for $G=S L_{2}$, as seen from the previous section.

Let $S$ be an affine algebraic subgroup scheme of $G$, and let $M$ be a $S$-module. For $g \in G$, one can consider the twisted module $M^{g}$ which is a $S^{\prime}=g^{-1} S g$-module. (cf. [26, I. 2.15]) In particular if $g$ normalizes $S$ then the twisted module $M^{g}$ becomes a $S$-module. Moreover, if $M$ is a $G$-module then $M^{g} \cong M$.

For $J \subseteq \Delta$, let $L_{J}$ be the Levi subgroup of $G$ determined by $J$. Set $X_{J}(T)_{+}=$ $\left\{\lambda \in X(T): 0 \leq\left\langle\lambda, \alpha^{\vee}\right\rangle\right.$ for all $\left.\alpha \in J\right\}$. For $\lambda \in X_{J}(T)_{+}$, one has a nonzero induced module $H_{J}^{0}(\lambda):=\operatorname{ind}_{L_{J} \cap B}^{L_{J}} \lambda$ with simple $L_{J}$-socle $L_{J}(\lambda)$. Moreover, one can define the Weyl module $V_{J}(\lambda)$ with head $L_{J}(\lambda)$. Let $T_{J}(\lambda)$ be the indecomposable $L_{J}$-tilting module of highest weight $\lambda$.

Following [28] (cf. [26, II. 5.21]) we have a weight space decomposition:

$$
H^{0}(\lambda)=\left(\bigoplus_{\nu \in \mathbb{Z} J} H^{0}(\lambda)_{\lambda-\nu}\right) \oplus M .
$$


where $M$ is the direct sum of all weight spaces $H^{0}(\lambda)_{\sigma}$ where $\sigma \neq \lambda-\nu$ for any $\nu \in \mathbb{Z} J$. Note that $H_{J}^{0}(\lambda)=\oplus_{\nu \in \mathbb{Z} J} H^{0}(\lambda)_{\lambda-\nu}$ and the aforementioned decomposition is $L_{J}$-stable. Therefore,

$$
\left.H^{0}(\lambda)\right|_{L_{J}} \cong H_{J}^{0}(\lambda) \oplus M
$$

Dually, we have that

$$
\left.V(\lambda)\right|_{L_{J}} \cong V_{J}(\lambda) \oplus M^{\prime}
$$

for some $L_{J}$-module $M^{\prime}$. The same argument works for the simple module $L(\lambda)$ and there is a decomposition:

$$
\left.L(\lambda)\right|_{L_{J}} \cong L_{J}(\lambda) \oplus N
$$

for some $L_{J}$-module $N$.

Observe that $L(\lambda)=\operatorname{soc}_{G}\left(H^{0}(\lambda)\right)$ which implies that $\operatorname{soc}_{L_{J}} L(\lambda) \subseteq \operatorname{soc}_{L_{J}}\left(H^{0}(\lambda)\right)$. Note that $L_{J}(\lambda)=\operatorname{soc}_{L_{J}}\left(H_{J}^{0}(\lambda)\right) \subseteq \operatorname{soc}_{L_{J}}\left(H^{0}(\lambda)\right)$. Moreover, $L_{J}(\lambda)$ appears as an $L_{J}$-composition factor of $L(\lambda)$ and $H^{0}(\lambda)$ with multiplicity one. Therefore, $L_{J}(\lambda)$ must appear in $\operatorname{soc}_{L_{J}} L(\lambda)$. By a dual argument, we can use the decomposition

$$
\left.V(\lambda)\right|_{L_{J}} \cong V_{J}(\lambda) \oplus N
$$

to show that $L_{J}(\lambda)$ appears in the head of $\left.L(\lambda)\right|_{L_{J}}$. The fact that $L_{J}(\lambda)$ has multiplicity one in $L(\lambda)$ now shows that $L_{J}(\lambda)$ is a $L_{J}$-direct summand of $L(\lambda)$.

Finally, we observe that $\left.T(\lambda)\right|_{L_{J}}$ is a tilting module for the Levi subgroup $L_{J}$ and we have that

for some $L_{J}$-tilting module $T$.

$$
\left.T(\lambda)\right|_{L_{J}} \cong T_{J}(\lambda) \oplus T
$$

We first show that the nontrivial induced modules and Weyl modules for groups of Lie rank greater than two are not endotrivial.

Theorem 8.1. Assume that $p>2$. If $\operatorname{rank} G=2$ (i.e., $\Phi=A_{2}, B_{2}$ or $G_{2}$ ), then the only induced module $H^{0}(\lambda)$ (resp. Weyl module $V(\lambda)$ ) which is endotrivial over $G_{r}$ is the trivial module.

Proof. We first note that if a module is endotrivial then the dual of the module is also endotrivial so the statement of the theorem for induced modules will imply the statement for Weyl modules.

Let us first consider the case when $\Phi=A_{2}$ with $p>2$. Let $\lambda=\left(\lambda_{1}, \lambda_{2}\right)$. Observe that if $\lambda_{1}$ or $\lambda_{2}$ has the form $n p^{r}-1$ then by Weyl's Dimension Formula, $p \mid \operatorname{dim} H^{0}(\lambda)$ thus $H^{0}(\lambda)$ cannot be endotrivial. So we can assume that neither $\lambda_{1}$ or $\lambda_{2}$ has the form $n p^{r}-1$. Let $J_{1}=\left\{\alpha_{1}\right\}$ and $J_{2}=\left\{\alpha_{2}\right\}$. Then we can consider the following decompositions:

$$
\begin{aligned}
& \left.H^{0}(\lambda)\right|_{L_{J_{1}}} \cong H_{J_{1}}^{0}(\lambda) \oplus M_{1}, \\
& \left.H^{0}(\lambda)\right|_{L_{J_{2}}} \cong H_{J_{2}}^{0}(\lambda) \oplus M_{2} .
\end{aligned}
$$

Set $w_{1}=s_{\alpha_{2}}$ and $w_{2}=s_{\alpha_{1}}$. Then $L=w_{1}\left(L_{J_{1}}\right)=w_{2}\left(L_{J_{2}}\right)$ where $L /[L, L]$ has type $A_{1}$ and contains the root subgroup $U_{\alpha_{1}+\alpha_{2}}$. The aforementioned decompositions can be twisted by $w_{1}$ (resp. $w_{2}$ ) to obtain

$$
\begin{aligned}
& \left.H^{0}(\lambda)\right|_{L} \cong H_{J_{1}}^{0}(\lambda)^{w_{1}} \oplus M_{1}^{w_{1}}, \\
& \left.H^{0}(\lambda)\right|_{L} \cong H_{J_{2}}^{0}(\lambda)^{w_{2}} \oplus M_{2}^{w_{2}} .
\end{aligned}
$$


Observe that $H_{J_{i}}^{0}(\lambda)^{w_{i}}$ for $i=1,2$ are indecomposable nonisomorphic $L$-module whose characters are not equal, unless $\lambda_{1}=\lambda_{2}=0$. Thus, $H_{J_{i}}^{0}(\lambda)^{w_{i}}$ for $i=1,2$ must appear as indecomposable $L$-summands of $\left.H^{0}(\lambda)\right|_{L}$. If $\left.H^{0}(\lambda)\right|_{G_{r}}$ is an endotrivial module, then $\left.H^{0}(\lambda)\right|_{L_{r}}$ is the direct sum of an endotrivial $L_{r}$-module and a projective $L_{r}$-module. But, $\left.H^{0}(\lambda)\right|_{L_{r}}$ contains summands $\left.H_{J_{i}}^{0}(\lambda)^{w_{i}}\right|_{L_{r}}$ for $i=1,2$, neither of which is projective since $\lambda_{i}$ is not of the form $n p^{r}-1$ for $i=1,2$.

For type $B_{2}$, note that $\lambda_{1}$ and $\lambda_{2}$ cannot both have the form $n p^{r}-1$. For otherwise, $p \mid \operatorname{dim} H^{0}(\lambda)$, and hence $H^{0}(\lambda)$ could not be endotrivial. Suppose that $\lambda_{1}$ is not a multiple of $p^{r}-1$. Let $w=s_{\alpha_{1}+\alpha_{2}}$ and $J=\left\{\alpha_{1}\right\}$. First note that

$$
\left.H^{0}(\lambda)\right|_{L_{J}} \cong H_{J}^{0}(\lambda) \oplus M
$$

Because $\alpha_{1}+\alpha_{2}$ is orthogonal to $\alpha_{1}, w\left(L_{J}\right)=L_{J}$, and $H^{0}(\lambda) \cong H^{0}(\lambda)^{w}$ as $G$ modules,

$$
\left.H^{0}(\lambda)\right|_{L_{J}} \cong H_{J}^{0}(\lambda)^{w} \oplus M^{w}
$$

The highest weight of $H_{J}^{0}(\lambda)^{w}$ is $w \lambda$. This is given by the formula

$$
s_{\alpha_{1}+\alpha_{2}} \lambda=\lambda-\left\langle\lambda,\left(\alpha_{1}+\alpha_{2}\right)^{\vee}\right\rangle\left(\alpha_{1}+\alpha_{2}\right)=\left(\lambda_{1},-\lambda_{1}-\lambda_{2}\right) .
$$

This demonstrates that the indecomposable $L_{J}$-modules $H_{J}^{0}(\lambda)$ and $H_{J}^{0}(\lambda)^{w}$ both appear as summands of $\left.H^{0}(\lambda)\right|_{L_{J}}$. But, if $\left.H^{0}(\lambda)\right|_{G_{r}}$ is endotrivial then $H^{0}(\lambda)_{L_{J}}$ can have at most one endotrivial direct summand which can only occur if $\lambda_{1}=\lambda_{2}=0$. A similar argument can be used for the case when $\lambda_{2}$ is not a multiple of $p^{r}-1$. In this case, one can take $J=\left\{\alpha_{2}\right\}$ with $w=s_{2 \alpha_{1}+\alpha_{2}}$, so that

$$
s_{2 \alpha_{1}+\alpha_{2}} \lambda=\left(-\lambda_{1}-2 \lambda_{2}, \lambda_{2}\right) \text {. }
$$

The argument for $\Phi=G_{2}$ follows the same line of reasoning as in the $B_{2}$-case with the existence of orthogonal roots. When $J=\left\{\alpha_{1}\right\}$ (resp. $J=\left\{\alpha_{2}\right\}$ ), take $w=s_{3 \alpha_{1}+2 \alpha_{2}}\left(\right.$ resp. $\left.w=s_{2 \alpha_{1}+\alpha_{2}}\right)$ and observe that

$$
\begin{gathered}
s_{3 \alpha_{1}+2 \alpha_{2}} \lambda=\left(\lambda_{1},-\lambda_{1}-\lambda_{2}\right), \\
s_{2 \alpha_{1}+\alpha_{2}} \lambda=\left(-\lambda_{1}-3 \lambda_{2}, \lambda_{2}\right) .
\end{gathered}
$$

Theorem 8.2. Suppose that $p>2$ and that $\operatorname{rank} G \geq 2$. Then the only induced module $H^{0}(\lambda)$ (resp. Weyl module $V(\lambda)$ ) which is endotrivial over $G_{r}$ is the trivial module.

Proof. It suffices to prove that statement for the induced modules $H^{0}(\lambda)$ with $\lambda=$ $\sum_{i=1}^{n} \lambda_{i} \omega_{i}$. Choose adjacent simple roots $\alpha_{i}, \alpha_{j}$ in the Dynkin diagram and set $J=\left\{\alpha_{i}, \alpha_{j}\right\} \subseteq \Delta$. Now

$$
\left.H^{0}(\lambda)\right|_{L_{J}} \cong H_{J}^{0}(\lambda) \oplus M
$$

Therefore, $H_{J}^{0}(\lambda)$ must be endotrivial over $\left(L_{J}\right)_{r}$. From the preceding theorem, we have $\lambda_{i}=\lambda_{j}=0$. Since this occurs for all pairs of adjacent simple roots, we conclude that $\lambda=0$.

The next result demonstrates that the restrictions of simple nontrivial $G$-modules for semisimple groups of rank larger that 1 cannot be endotrivial modules for $G_{r}$. Note that there is no restriction on the prime in this result. 
Theorem 8.3. Suppose that $\operatorname{rank} G \geq 2$. Then the only irreducible $G$-module $L(\lambda)$ which is endotrivial over $G_{r}$ is the trivial module.

Proof. Recall first from the proof of Corollary 6.4(a), that we can assume that $\lambda \in X_{1}(T)$. Now let $J=\left\{\alpha_{i}\right\}$ where $\alpha_{i} \in \Delta$. Then

$$
\left.L(\lambda)\right|_{\left(L_{J}\right)_{1}} \cong L_{J}(\lambda) \oplus M
$$

where $L_{J}(\lambda)$ must either be endotrivial or projective. Our analysis of the $A_{1}$ case implies that $\lambda_{i}=0, p-2$ or $p-1$.

One can now use the rank 2 argument involving twisting the decomposition

$$
\left.L(\lambda)\right|_{L_{J}} \cong L_{J}(\lambda) \oplus M
$$

via Weyl group elements given for induced modules and Weyl modules in the proof of Theorem 8.1 to show that the endotriviality of $\left.L(\lambda)\right|_{G_{r}}$ implies that $\lambda=0$ for $p \geq 3$. The remaining $p=2$ case for $\Phi=A_{2}$ (resp. $\left.B_{2}, G_{2}\right)$ can be handled as follows. We can enumerate the different possibilities for $\lambda \in X_{1}(T)$. The module $L(1,0), L(0,1), L(1,1)$ have dimensions which are never congruent to \pm 1 , modulo $2^{3 r}$ (resp. $2^{4 r}, 2^{6 r}$ ), thus cannot be endotrivial over $G_{r}$.

Finally, one can use the argument presented in the proof of Theorem 8.2 to handle the general case by restricting to irreducible rank 2 subroot systems of $\Phi$ to show that $\lambda=0$.

We now show that endotrivial modules rarely occur as restrictions of tilting modules. Note that if a tilting module $T(\lambda)$, which satisfies the condition that $-w_{0} \lambda=\lambda$, were endotrivial, then this module would generate a subgroup of order 2 inside of $T\left(G_{r}\right)$ because $T(\lambda) \cong T(\lambda)^{*}$.

Theorem 8.4. Let $\lambda \in X(T)_{+}$and let $T(\lambda)$ be the corresponding indecomposable tilting module. Suppose that $\operatorname{rank} G \geq 2$ with $p \geq h$. Then the only indecomposable tilting module $T(\lambda)$ which is endotrivial over $G_{r}$ is the trivial module.

Proof. For the rank two cases, one can now use the argument given in Theorem 8.1 and the rank one analysis above, to prove that if $\left.T(\lambda)\right|_{G_{r}}$ is endotrivial for $\Phi=A_{2}$, $B_{2}$ or $G_{2}$, then $\lambda=0$.

For the general case, we first note that if $p \geq h$, then the assumption that $\left.T(\lambda)\right|_{G_{r}}$ is an endotrivial module implies that $0 \leq \lambda_{j} \leq p-2$ for $j=1,2, \ldots, n$. If $\lambda_{j}>p-1$ then $p \mid T(\lambda)$ by [10, Theorem 5.3] and $\left.T(\lambda)\right|_{G_{r}}$ is not endotrivial. Choose adjacent simple roots $\alpha_{i}, \alpha_{j}$ in the Dynkin diagram and set $J=\left\{\alpha_{i}, \alpha_{j}\right\} \subseteq \Delta$. Now

$$
\left.T(\lambda)\right|_{L_{J}} \cong T_{J}(\lambda) \oplus M
$$

Since $0 \geq \lambda_{i}, \lambda_{j}<p-2, T_{J}(\lambda)$ is not projective (cf. [26, E.8. Lemma]), thus must be endotrivial so $\lambda_{i}=\lambda_{j}=0$. As this works for any pair of adjacent roots, we have that $\lambda=0$.

\section{Connection with Picard Groups}

In this section we indicate how our work fits in with the triangular geometry introduced by Balmer. Given a finite dimensional cocommutative Hopf algebra $A$, we denote the stable module category by $\mathcal{K}=A$-stab. This is the quotient of 
the category of $A$-modules by maps that factor through projective modules. The category $\mathcal{K}$ is a tensor triangulated category and thus inherits a "tensor triangulated geometry" as introduced by Balmer [2].

Let $\operatorname{Spc}(\mathcal{K})$ be the spectrum of $\mathcal{K}$. As pointed out in [3], $\operatorname{Spc}(\mathcal{K})$ has a sheaf of commutative rings $\mathcal{O}_{\mathcal{K}}$ which makes it a locally ringed spaced. This locally ringed space is denoted by $\operatorname{Spec}(\mathcal{K})$.

In [2, Theorem 6.3(b)], it is shown that

$$
\operatorname{Spec}(A-\operatorname{stab}) \cong \operatorname{Proj}\left(\mathcal{V}_{A}(k)\right) \cong \operatorname{Proj}\left(\mathrm{H}^{\bullet}(A, k)\right) .
$$

By interpreting Balmer's construction [3, Construction 2.6] in our setting we have group homomorphisms.

$$
\operatorname{Pic}\left(\operatorname{Proj}\left(\mathcal{V}_{A}(k)\right) \stackrel{\alpha}{\leftarrow} \operatorname{Pic}_{\text {loctr. }}(\mathcal{K}) \hookrightarrow T(A) .\right.
$$

where Pic denote the group of line bundles and $T(A)$ is the group of endotrivial modules. Moreover, we have the following theorem.

Theorem 9.1. Let $A$ be a finite dimensional cocommutative Hopf algebra. Then there exists a monomorphism of groups

$$
\beta: \operatorname{Pic}\left(\operatorname{Proj}\left(\mathcal{V}_{A}(k)\right) \otimes_{\mathbb{Z}} \mathbb{Z}[1 / p] \hookrightarrow T(A) \otimes_{\mathbb{Z}} \mathbb{Z}[1 / p] .\right.
$$

We remark that Balmer proves using the gluing techniques of Balmer-Favi [5] and Balmer-Benson-Carlson [4] that $\beta$ becomes an isomorphism when $A=k G$ where $G$ is a finite group after tensoring by $\mathbb{Q}$ (cf. [3, Theorem 4.7]). It is still open if this holds for arbitrary finite group schemes.

Let us look at two examples in the setting of infinitesimal group schemes.

Example 9.2. Let $A=\operatorname{Dist}\left(G_{1}\right)$ where $G=S L_{2}$. We have seen that $T(A)=$ $\mathbb{Z} \oplus \mathbb{Z} / 2 \mathbb{Z}$. On the other hand, if $p \geq 3$, then $\mathcal{V}_{A}(k) \cong \mathcal{N}$ where $\mathcal{N}$ is the set of $2 \times 2$ nilpotent matrices. In this case, $\operatorname{Pic}(\operatorname{Proj}(\mathcal{N})) \cong \mathbb{Z}$. This shows that the Picard group and the endotrivial groups do not identify themselves on the integral level.

Example 9.3. Let $A=\operatorname{Dist}\left(U_{1}\right)$ where $U$ is the unipotent radical of a Borel subgroup. If $p \neq 2$ when the underlying root system is of type $A_{2}$ or $B_{2}$ then $T\left(U_{1}\right) \cong \mathbb{Z}$. We know that $\operatorname{Pic}\left(\operatorname{Proj}\left(\mathcal{V}_{U_{1}}(k)\right)\right)$ has rank at least one. The theorem above demonstrates that $\operatorname{rank} \operatorname{Pic}\left(\operatorname{Proj}\left(\mathcal{V}_{U_{1}}(k)\right)\right)=1$.

\section{Open Problems:}

We conclude this paper by presenting several open questions which the authors view as worthy of further investigation.

Let $P$ be a projective indecomposable $G_{r}$-module. It has been a long standing conjecture that the $G_{r}$-action of $P$ should lift to a rational action of $G$. Ballard showed that this holds when $p \geq 3(h-1)$ and Jantzen proved this when $p \geq 2(h-1)$. For a discussion of this problem, see [26, II. 11.11]. This motivates our first question.

(1) Let $G$ be a reductive algebraic group and let $M$ be an endotrivial $G_{r}$-module. Does $M$ lift to a $G$-structure? 
We have seen that in most cases our computations show that $T\left(H_{r}\right) \cong \mathbb{Z}$ where $H$ is an affine algebraic group scheme. This provides some basis for asking the following two questions:

(2) Let $H$ be an arbitrary finite group scheme. Is $T(H)$ finitely generated? For finite groups this was first proved by Puig [27], but also follows from the the classification of endotrivial modules over $p$-groups [16].

The final two questions entail a finding a more detailed description of our computations in Sections 5-8.

(3) Suppose that $U$ is the unipotent radical of the Borel subgroup of a semisimple algebraic group $G$. For $r \geq 2$, does $A=\operatorname{Dist}\left(U_{r}\right)$ satisfy Hypothesis 4.5, or more generally is $T\left(U_{r}\right)$ generated by $\Omega(k)$ ?

(4) Determine the structure of $T\left(\left(S L_{2}\right)_{r}\right)$ and in general $T\left(G_{r}\right)$ where $G$ is a reductive algebraic group scheme. This is the first step in solving the more general question of finding $T(G)$ for $G$ a finite group scheme.

\section{REFERENCES}

[1] J. Alperin, A construction of endo-permutation modules, J. Group Theory, 4 (2001), $3-10$.

[2] P. Balmer, The spectrum of prime ideals in tensor triangulated categories, J. Reine Angew. Math., 588 (2005), 149-168.

[3] P. Balmer, Picard groups in triangular geometry and applications to modular representation theory, preprint, (2009).

[4] P. Balmer, D. Benson and J. Carlson, Gluing representations via idempotent modules and constructing endotrivial modules, J. Pure Appl. Algebra, 213 (2009), 173 -193.

[5] P. Balmer and G. Favi, Gluing techniques in triangular geometry, Quarterly J. Math., 58 (2007), 415-441.

[6] S. Bouc, The Dade group of a p-group, Invent. Math., 164 (2006), 189-231.

[7] M. Bremner, R. Moody, J. Patera, Tables of dominant weight multiplicities for representations of simple Lie algebras, Monographs and Textbooks in Pure and Applied Mathematics, 90. Marcel Dekker, Inc., New York, 1985.

[8] J. Carlson, E. Friedlander and J. Pevtsova, Modules of constant Jordan type, J. Reine Angew. Math., 2008 (2008), 191-234.

[9] J. Carlson, D. Hemmer and N. Mazza, The group of endotrivial modules for the symmetric and alternating groups, Proc. Edinburgh Math. Soc. (to appear).

[10] J. Carlson, Z. Lin, D. Nakano, B. Parshall, The restricted nullcone, Cont. Math., 325 (2003), 51-75.

[11] J. Carlson, D. Nakano, K. Peters, On the vanishing of extensions of modules over reduced enveloping algebras, Math. Ann., 302 (1995), 541-560.

[12] J. Carlson, N. Mazza and D. Nakano, Endotrivial modules for finite groups of Lie type, J. Reine Angew. Math., 595 (2006), 284-306.

[13] J. Carlson, N. Mazza and D. Nakano, Endotrivial modules for the symmetric group, Proc. Edinburgh Math. Soc., 51 (2008), 1-22.

[14] J. Carlson and J. Thévenaz, Torsion endotrivial modules, Algebras and Representation Theory, 3 (2000), 303-335.

[15] J. Carlson and J. Thévenaz, The classification of torsion endo-trivial modules, Annals of Math., (2) 165 (2005), 823-883. 
[16] J. Carlson and J. Thévenaz, The classification of endo-trivial modules, Invent. Math., 158 (2004), 389-411.

[17] E. Dade, Endo-permutation modules over p-groups I, Annals of Math., 107 (1978), 459494.

[18] E. Dade, Endo-permutation modules over p-groups II, Annals of Math., 108 (1978), 317-346.

[19] E. Dade, Algebraically rigid modules, Representation theory, II (Proc. Second Internat. Conf., Carleton Univ., Ottawa, Ont., 1979), pp. 195-215, Lecture Notes in Math., 832, Springer, Berlin, 1980.

[20] E. Friedlander, B. Parshall, Support varieties for restricted Lie algebras, Invent. Math., 86 (1986), 553-562.

[21] E. Friedlander, J. Pevtsova, Representation-theoretic support spaces for finite group schemes, Amer. J. Math., 127 (2005), 379-420.

[22] E. Friedlander, J. Pevtsova, П-supports for modules for finite group schemes, Duke. Math. J., 107 (2007), 317-368.

[23] E. Friedlander, J. Pevtsova, A Suslin, Generic and maximal Jordan types, Invent. Math (to appear).

[24] J. Humphreys, Introduction to Lie algebras and representation theory, Graduate Texts in Mathematics, Vol. 9., Springer-Verlag, New York-Berlin, 1972.

[25] J. Jantzen, Kohomologie von p-Lie Algebren und nilpotente Elemente, Abh. Math. Sem. Univ. Hamburg 56 (1986), 191-219.

[26] J. Jantzen, Representations of algebraic groups, Second Edition, American Mathematical Society, Providence R.I., 2003.

[27] L. Puig, Affirmative answer to a question of Feit, J. Algebra, 131 (1990), 513-526.

[28] S. Smith, Irreducible modules and parabolic subgroups, J. Algebra, 75 (1982), 286-289.

Department of Mathematics, University of Georgia, Athens, Georgia 30602, USA

E-mail address: jfc@math.uga.edu

Department of Mathematics, University of Georgia, Athens, Georgia 30602, USA

E-mail address: nakano@math.uga.edu 